\title{
Adjoint-Based Mesh Adaptation and Shape Optimization for Simulations with Propulsion
}

\author{
Marian Nemec* \\ NASA Ames Research Center, Moffett Field, CA 94035, USA \\ David L. Rodriguez ${ }^{\dagger}$ \\ Science \&3 Technology Corp., Moffett Field, CA 94035, USA \\ Michael J. Aftosmis $\ddagger$ \\ NASA Ames Research Center, Moffett Field, CA 94035, USA
}

\begin{abstract}
We demonstrate a well-posed formulation of permeable boundary conditions and massflow-rate functionals for adjoint-based mesh refinement and shape optimization governed by the steady Euler equations. The boundary conditions are used to model propulsionsystem effects of inlets and nozzles. A two-shock diffuser with an analytic solution is used to verify the implementation. Numerical examples show that the adjoint solution is smooth at the boundary, indicating that the discretization is adjoint consistent when exit pressure is specified at subsonic outflow, and stagnation temperature and pressure at subsonic inflow. The results focus on improving simulation techniques for low-boom aircraft analysis and design. By including mass-flow-rate outputs, we obtain reliable estimates of engine flow rates concurrently with nearfield pressure signatures without increasing simulation cost. We also demonstrate the importance of mass-flow-rate constraints in shape optimization by examining trade-offs between maximizing performance of a shrouded supersonic nozzle and minimizing shocks in its nearfield.
\end{abstract}

\section{Introduction}

$\mathrm{T}$ The modeling of propulsion systems can be important when predicting and optimizing the external aerodynamics of air vehicles. For example, simulations with active inlets and exhaust plumes are required to accurately capture the complex near-body shock system of low-boom supersonic aircraft to predict both aerodynamic performance and ground noise carpets. ${ }^{1-3}$ Similarly, computational studies of high-bypass fanjet installations must accurately resolve the propulsive stream-tube to capture the changes in the induced drag due to the non-planar lifting system and the interference effects of transonic wave drag. ${ }^{4,5}$ Another important example is efficient simulation of helicopters and multi-rotor vehicles, where capturing the rotor interference effects is essential to predicting aerodynamic performance. ${ }^{6}$

In fixed-wing applications, specialized permeable boundary conditions ${ }^{7}$ and actuator zones ${ }^{8}$ are typically used to model the propulsion system. Moreover, specialized outputs, such as those involving mass flow rates and stagnation pressure, ${ }^{9-11}$ are required to characterize the aerodynamic performance of powered configurations. These add various challenges to the simulation - most notably, appropriate meshing of the propulsive stream-tube and exhaust plumes to control the discretization error in the quantities of interest. While the method of adjoint weighted residuals has proven to be exceptionally effective for estimating error in aerodynamic forces and moments, ${ }^{12}$ analysis and application of the method in simulations involving engine boundary conditions and general boundary outputs has been largely unexplored. In gradient-based aerodynamic shape optimization, by contrast, the adjoint method has been successfully applied to many powered configurations. ${ }^{8,11,13}$ The adjoint equation solved in both settings is the same, but the computation

*Aerospace Engineer, Computational Aerosciences Branch, MS 258-5; marian.nemec@nasa.gov, Senior Member AIAA.

†Senior Research Scientist, Computational Aerosciences Branch; david.l.rodriguez@nasa.gov, Senior Member AIAA.

$¥$ Aerospace Engineer, Computational Aerosciences Branch; michael.aftosmis@nasa.gov, Associate Fellow AIAA. 
of the objective function gradient is perhaps less sensitive to issues regarding adjoint consistency, especially when using the discrete adjoint approach, leading to more widespread use. The work of Ordaz et al. ${ }^{10}$ on engine inlet distortion focuses on adjoint-based optimization, but also demonstrates progress toward adjoint-based mesh adaptation for stagnation-pressure outputs.

The purpose of this paper is twofold. First, we apply the method of adjoint weighted residuals in simulations of powered configurations with adaptive mesh refinement, where the output of interest is the mass flow rate. Second, we reuse the adjoint solution for gradient computations to enable shape optimization in problems with mass-flow-rate objectives and constraints. In recent work, ${ }^{14}$ we formulated several boundary conditions applicable to inlets, turbines and nozzle plenums. While these boundary conditions provide only a highly simplified model of an actual engine, they are especially effective when full engine details are not available or not important, and when modeling secondary flow paths. The boundary conditions are implemented in an adjoint-based mesh adaptation procedure ${ }^{15}$ for pressure-based outputs, e.g., force and moment coefficients. The adaptive refinement is part of a gradient-based shape optimization framework ${ }^{16}$ to control the level of discretization error in the design space. The optimization starts on a coarse mesh that is progressively refined as the design improves, resulting in a faster optimization and greater confidence in the final design. The present work is an important step toward extending this framework to handle propulsion-airframe integration problems.

Of particular interest are analysis and design problems related to low-boom supersonic flight. We proceed by analyzing the linearization of the boundary conditions and the mass-flow-rate functional, and present several numerical experiments to examine the behavior of the adjoint near the permeable boundary. A verification case is constructed to test the accuracy of the discretization error estimate and the effectiveness of the mesh adaptation at annihilating discretization error. We demonstrate the approach on a practical simulation based on a detailed prototype of NASA's X-59 Low-Boom Flight Demonstrator. This aircraft model features several outflow and inflow boundary conditions to capture the effects of the main engine and secondary flow paths. Efficient simulation techniques for predicting noise from low-boom configurations have been the focus of the first and second AIAA Sonic-Boom Prediction Workshops. ${ }^{17-19}$ We extend these techniques to powered configurations by demonstrating the importance of including mass-flow-rate outputs to predict aerodynamic performance more accurately without compromising the nearfield pressure signature. Thereafter, we present two optimization test cases that involve mass-flow-rate steering and constraints.

\section{Governing Equations and Outputs of Interest}

\section{A. Flow Equations}

We solve the three-dimensional Euler equations governing compressible flow of a perfect gas. For a finite region of space with volume $V$ and surface area $A$, the integral form of the Euler equations is given by

$$
\frac{\mathrm{d}}{\mathrm{d} t} \int_{V} \mathbf{Q} \mathrm{d} V+\oint_{A} \mathbf{F} \cdot \hat{\mathbf{n}} \mathrm{d} A=\mathbf{0}
$$

where $\mathbf{Q}=[\rho, \rho \mathbf{U}, \rho E]^{\mathrm{T}}=[\rho, \rho u, \rho v, \rho w, \rho E]^{\mathrm{T}}$ are the non-dimensional conserved variables, and

$$
\mathbf{F} \cdot \hat{\mathbf{n}}=\left[\begin{array}{c}
\rho U_{\mathrm{n}} \\
\rho U_{\mathrm{n}} \mathbf{U}+p \hat{\mathbf{n}} \\
(\rho E+p) U_{\mathrm{n}}
\end{array}\right]
$$

where $U_{\mathrm{n}}=\mathbf{U} \cdot \hat{\mathbf{n}}$ and $\hat{\mathbf{n}}$ is the outward facing unit normal.

\section{B. Output Functionals}

The primary output of interest is the mass flow rate through a user-specified region of the vehicle's wetted surface $\mathcal{B}$ where permeable boundary conditions are applied

$$
\mathcal{J}_{\dot{m}}=\int_{\mathcal{B}} \rho U_{\mathrm{n}} \mathrm{d} A
$$


Additional outputs are aerodynamic performance coefficients, such as coefficients of lift and drag, computed over all or a portion of the wetted surface $\mathcal{W}$

$$
\mathcal{J}_{a}=\frac{1}{q_{\infty} A_{\text {ref }}} \int_{\mathcal{W}}(\hat{\mathbf{n}} \cdot \hat{\boldsymbol{\xi}})\left(p-p_{\infty}\right) \mathrm{d} A
$$

where $q_{\infty}$ is the freestream dynamic pressure, $A_{\text {ref }}$ is the reference area, $p_{\infty}$ is the freestream pressure and $\hat{\boldsymbol{\xi}}$ is the appropriate projection for the coefficient of interest, e.g., $\hat{\boldsymbol{\xi}} \perp \mathbf{V}_{\infty}$ for lift. Lastly, the output may also involve off-body sensors, such as pressure-based line sensors that are frequently used in sonic-boom simulations

$$
\mathcal{J}_{l}=c \int_{0}^{L}\left(\frac{p-p_{\infty}}{p_{\infty}}\right)^{n} \omega(\ell, L) \mathrm{d} \ell
$$

where $L$ is the length of the line, $n$ is a user specified exponent (usually 1 or 2), and the constant $c$ and function $\omega$ are used when an equivalent area distribution or custom weights are desired.

\section{Numerical Method}

Equation 1 is discretized on a multilevel Cartesian mesh with embedded boundaries. The mesh consists of regular Cartesian hexahedra everywhere, except for a layer of bodyintersecting cells, or cut cells, adjacent to the wetted surface of the geometry, as illustrated in Fig. 1. The spatial discretization uses a cell-centered, second-order accurate finite volume method, resulting in a system of equations

$$
\mathbf{R}_{H}\left(\mathbf{Q}_{H}\right)=0
$$

evaluated on a mesh with an average cell size $H$, which we call the working mesh. The vector $\mathbf{Q}_{H}=\left[\bar{Q}_{1}, \bar{Q}_{2}, \ldots, \bar{Q}_{N}\right]^{\mathrm{T}}$ is the discrete solution vector of the cell-averaged values for all $N$ cells of the mesh. The flux-vector splitting approach of van Leer $^{20}$ is used. Steady-state flow solutions are obtained using a five-stage Runge-Kutta scheme with local timestepping, multigrid, and a domain decomposition scheme for parallel computing. ${ }^{21-23}$

All boundary conditions are enforced weakly. At slip walls,

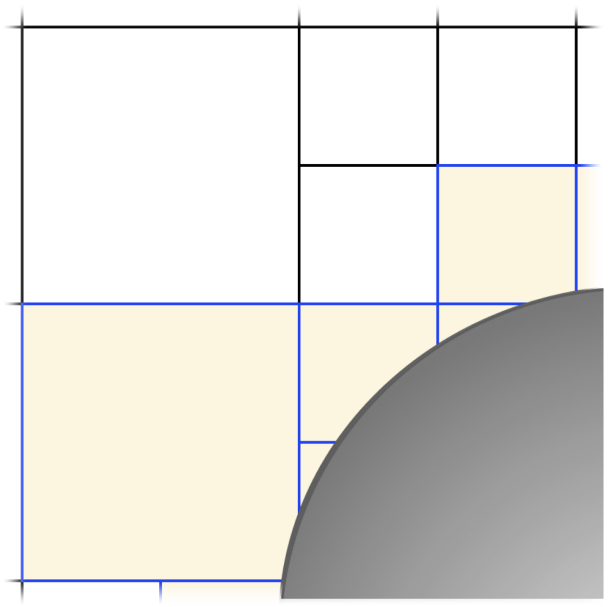

Figure 1: Multilevel Cartesian mesh in twodimensions with a cut-cell boundary. Adjacent cells cannot exceed 2:1 ratio. zero normal velocity, $U_{\mathrm{n}}=0$, simplifies Eq. 2 to the familiar $\mathbf{F} \cdot \hat{\mathbf{n}}=[0, p \hat{\mathbf{n}}, 0]^{\mathrm{T}}$. At permeable boundaries, we use the one-dimensional Riemann invariants

$$
\begin{aligned}
\Lambda & =p / \rho^{\gamma} \\
\Lambda^{+} & =U_{\mathrm{n}}+\frac{2 a}{\gamma-1} \\
\Lambda^{-} & =U_{\mathrm{n}}-\frac{2 a}{\gamma-1}
\end{aligned}
$$

to construct a boundary state $\mathbf{q}_{b}=\left[\rho_{b}, u_{b}, v_{b}, w_{b}, p_{b}\right]^{\mathrm{T}}$ to evaluate the boundary flux $\mathbf{F}\left(\mathbf{q}_{b}\right) \cdot \hat{\mathbf{n}}$ via Eq. 2 . We use primitive variables to simplify the presentation. The full implementation and discussion of these boundary conditions is given by Rodriguez et al. ${ }^{14}$ extending earlier work by Pandya et al. ${ }^{24}$ Here we give only the main results to set the stage for discussing the corresponding adjoint, or dual, boundary conditions in Section V.

For subsonic outflow through the wetted surface, e.g., when simulating engine inlets, the boundary state is constructed from one user-specified quantity, either exit pressure $p_{\text {set }}$ or normal velocity $U_{\mathrm{n}, \mathrm{set}}$. All other quantities are taken from the interior, i.e., from within the computational domain. For example, assuming flow in the positive $x$-direction and $\hat{\mathbf{n}}$ aligned with the positive $x$-coordinate, the outgoing Riemann invariants are $\Lambda$ and $\Lambda^{+}$. When we specify exit pressure $p_{\text {set }}$, the outgoing invariants are used along with preservation 
of tangential velocity to determine the boundary state

$$
\mathbf{q}_{b}=\left[\begin{array}{c}
\rho_{b} \\
\mathbf{U}_{b} \\
p_{b}
\end{array}\right]=\left[\begin{array}{c}
\rho\left(\frac{p_{\text {set }}}{p}\right)^{\frac{1}{\gamma}} \\
\left(\Lambda^{+}-\frac{2}{\gamma-1} a_{b}\right) \hat{\mathbf{n}}+\left(\mathbf{U}-U_{\mathrm{n}} \hat{\mathbf{n}}\right) \\
p_{\text {set }}
\end{array}\right]
$$

where $a_{b}=\sqrt{\frac{\gamma}{\rho}\left(p_{\text {set }}^{\frac{\gamma-1}{\gamma}}\right) p^{\frac{1}{\gamma}}}$. Similarly, when normal exit velocity is specified the boundary state is given by

$$
\mathbf{q}_{b}=\left[\begin{array}{c}
\rho_{b} \\
\mathbf{U}_{b} \\
p_{b}
\end{array}\right]=\left[\begin{array}{c}
\left(\frac{a_{b}^{2}}{\gamma \Lambda}\right)^{\frac{1}{\gamma-1}} \\
U_{\mathrm{n}, \text { set }} \hat{\mathbf{n}}+\left(\mathbf{U}-U_{\mathrm{n}} \hat{\mathbf{n}}\right) \\
\frac{\rho_{b} a_{b}^{2}}{\gamma}
\end{array}\right]
$$

where we redefine $a_{b}=\frac{\gamma-1}{2}\left(\Lambda^{+}-U_{\mathrm{n}, \text { set }}\right)$.

For subsonic inflow through the wetted surface, e.g., nozzle or plume simulations, the user specifies two quantities corresponding to the two incoming invariants, $\Lambda$ and $\Lambda^{+}$. The specified quantities are stagnation temperature $T_{\mathrm{t}, \mathrm{set}}$ and either stagnation pressure $p_{\mathrm{t}, \mathrm{set}}$ or the mass flow rate $\dot{m}_{\text {set }}$. These are frequently used quantities in engineering problems. In addition, zero tangential velocity is assumed. When total pressure is specified, we use $\Lambda^{-}$in conjunction with total enthalpy to determine the speed of sound at the boundary. This expression is complicated because it involves the solution of a quadratic equation. For our purposes, it is sufficient to write the boundary state as

$$
\mathbf{q}_{b}=\left[\begin{array}{c}
\rho_{b} \\
\mathbf{U}_{b} \\
p_{b}
\end{array}\right]=\left[\begin{array}{c}
\frac{\gamma}{T_{\mathrm{t}, \mathrm{set}}} p_{b}^{\frac{1}{\gamma}} p_{\mathrm{t}, \mathrm{set}}^{\frac{\gamma-1}{\gamma}} \\
V \hat{\mathbf{n}} \\
p_{\mathrm{t}, \mathrm{set}}\left(1+\frac{\gamma-1}{2} M_{b}^{2}\right)^{\frac{\gamma}{1-\gamma}}
\end{array}\right]
$$

where $V=\Lambda^{+}-\frac{2 a_{b}}{\gamma-1}$ and $M_{b}=V / a_{b}$.

When mass flow rate is specified, the Riemann invariants are not used explicitly. The boundary density is taken from the interior, which sets the normal velocity, and the boundary pressure is determined via the energy equation

$$
\mathbf{q}_{b}=\left[\begin{array}{c}
\rho_{b} \\
\mathbf{U}_{b} \\
p_{b}
\end{array}\right]=\left[\begin{array}{c}
\rho \\
\frac{\dot{m}_{\mathrm{set}}}{\rho A} \hat{\mathbf{n}} \\
\frac{\rho}{\gamma}\left(T_{\mathrm{t}, \mathrm{set}}-\frac{\gamma-1}{2}\left(\frac{\dot{m}_{\mathrm{set}}}{\rho A}\right)^{2}\right)
\end{array}\right]
$$

where $A$ is the area of the permeable surface. Substituting the $\mathbf{q}_{b}$ states into Eq. 2 gives the flux across the boundary face that is used in the evaluation of the residual.

\section{Discretization Error Estimate}

Let $J_{H}\left(\mathbf{Q}_{H}\right)$ denote an approximation of the output functional, Eqs. 3-5, on the working mesh. To approximate the discretization error

$$
\mathcal{E}=\left|\mathcal{J}(\mathbf{Q})-J_{H}\left(\mathbf{Q}_{H}\right)\right|
$$

we consider isotropic refinement of the working mesh to obtain an embedded mesh with average cell size $h$ containing approximately $8 N$ cells (in three dimensions). The goal is to affordably estimate the error relative to the embedded mesh

$$
e=\left|J_{h}\left(\mathbf{Q}_{h}\right)-J_{H}\left(\mathbf{Q}_{H}\right)\right|
$$


and then rely on asymptotic analysis ${ }^{25}$ to determine the total discretization error

$$
\mathcal{E}=\left(1+\frac{1}{r^{p}-1}\right) e
$$

where $p$ is the order of accuracy of the spatial discretization and $r$ is the refinement ratio, $H / h$. For example, for a first-order scheme, $p=1$, and uniform refinement, $r=2$, Eq. 16 reduces to $\mathcal{E}=2 e$.

Our approach to estimate $J_{h}\left(\mathbf{Q}_{h}\right)$ follows the work of Venditti and Darmofal. ${ }^{26}$ The details of the implementation are given in Ref. 15. Summarizing briefly, the expression for the functional on the embedded mesh is given by

$$
J_{h}\left(\mathbf{Q}_{h}\right) \approx J_{h}\left(\mathbf{Q}_{\mathbf{L}}\right)-\underbrace{\left(\boldsymbol{\psi}_{h}^{H}\right)^{\mathrm{T}} \mathbf{R}_{h}\left(\mathbf{Q}_{\mathbf{L}}\right)}_{\text {Adjoint Correction }}-\underbrace{\left(\boldsymbol{\psi}_{h}-\boldsymbol{\psi}_{h}^{H}\right)^{\mathrm{T}} \mathbf{R}_{h}\left(\mathbf{Q}_{\mathbf{L}}\right)}_{\text {Remaining Error }}
$$

with $\mathbf{Q}_{\mathbf{L}}=\mathbf{P}_{\mathrm{L}} \mathbf{Q}_{H}$, where $\mathbf{P}_{\mathrm{L}}$ represents a linear prolongation operator, and $\boldsymbol{\psi}_{h}^{H}$ denotes a reconstruction of the adjoint variables from the working mesh to the embedded mesh. We approximate $\boldsymbol{\psi}_{h}$ in Eq. 17 with a triquadratic interpolant, $\boldsymbol{\psi}_{\mathrm{TQ}}$, and $\boldsymbol{\psi}_{h}^{H}$ with a trilinear interpolant, $\boldsymbol{\psi}_{\mathrm{TL}}$. The adjoint variables satisfy the following linear system of equations on the working mesh

$$
\left[\frac{\partial \mathbf{R}_{H}\left(\mathbf{Q}_{H}\right)}{\partial \mathbf{Q}_{H}}\right]^{\mathrm{T}} \psi_{H}=\frac{\partial J_{H}\left(\mathbf{Q}_{H}\right)^{\mathrm{T}}}{\partial \mathbf{Q}_{H}}
$$

To obtain a computable error estimate, we split Eq. 17 into an estimate for the corrected functional

$$
J_{c}=J_{h}\left(\mathbf{Q}_{\mathrm{L}}\right)-\boldsymbol{\psi}_{\mathrm{TQ}}^{\mathrm{T}} \mathbf{R}_{h}\left(\mathbf{Q}_{\mathrm{L}}\right)
$$

and a cell-wise estimate of the remaining error in each cell of the working mesh

$$
\boldsymbol{\eta}_{H}=\sum_{j \in \mathrm{V}_{i}}\left(\boldsymbol{\psi}_{\mathrm{TQ}}-\boldsymbol{\psi}_{\mathrm{TL}}\right)^{\mathrm{T}} \mathbf{R}_{h}\left(\mathbf{Q}_{\mathrm{L}}\right)_{j}
$$

where $j$ denotes the $j^{\text {th }}$ child of parent cell $\mathrm{V}_{i}$ and $\boldsymbol{\eta}_{H}=\left[\eta_{1}, \eta_{2}, \ldots, \eta_{N}\right]^{\mathrm{T}}$. To define a local quantity suitable for driving adaptive mesh refinement, the remaining error, Eq. 20, is localized to form a cell-wise error indicator $|\boldsymbol{\eta}|_{H}=\left[\left|\eta_{1}\right|,\left|\eta_{2}\right|, \ldots,\left|\eta_{N}\right|\right]^{\mathrm{T}}$. Substituting Eq. 19 into Eq. 15 gives an estimate of the relative error

$$
e \approx\left|J_{c}-J_{H}\left(\mathbf{Q}_{H}\right)\right|
$$

without solving on the embedded mesh.

\section{Adjoint Boundary Conditions}

An important step in the error estimation procedure is the formation and solution of the discrete adjoint equation (Eq. 18). This large linear system is wholly determined by the discrete flow residual, which includes the boundary conditions described in Section III, and the user-selected output. The system must be nonsingular and its solution should converge in the fine mesh limit to the solution of the continuous adjoint equation obtained by linearizing Eqs. 1-5 directly. Otherwise the error estimate, Eq. 21, may not be reliable. In particular, adjoint inconsistent formulations can generate spurious oscillations near the boundary, ${ }^{27-29}$ which pollute the reconstruction of $\boldsymbol{\psi}_{\mathrm{TQ}}$ and $\boldsymbol{\psi}_{\mathrm{TL}}$. We focus on the adjoint consistency of the permeable boundary conditions only; discussion regarding the linearization of the interior operators and the far-field boundaries, as well as the adjoint solution methodology can be found in Ref. 15.

The contribution to the adjoint system from a wall boundary face can be written as

$$
\frac{\partial \mathbf{q}_{b}{ }^{\mathrm{T}}}{\partial \mathbf{q}}{\frac{\partial(\mathbf{F} \cdot \hat{\mathbf{n}})}{\partial \mathbf{q}_{b}}}^{\mathrm{T}} \boldsymbol{\psi}={\frac{\partial J_{\dot{m}}}{\partial \mathbf{q}}}^{\mathrm{T}}
$$

where we used primitive variables to simplify the analysis and

$$
\frac{\partial J_{\dot{m}}}{\partial \mathbf{q}}=\left[\begin{array}{c}
U_{n} \\
\rho \hat{\mathbf{n}} \\
0
\end{array}\right]
$$




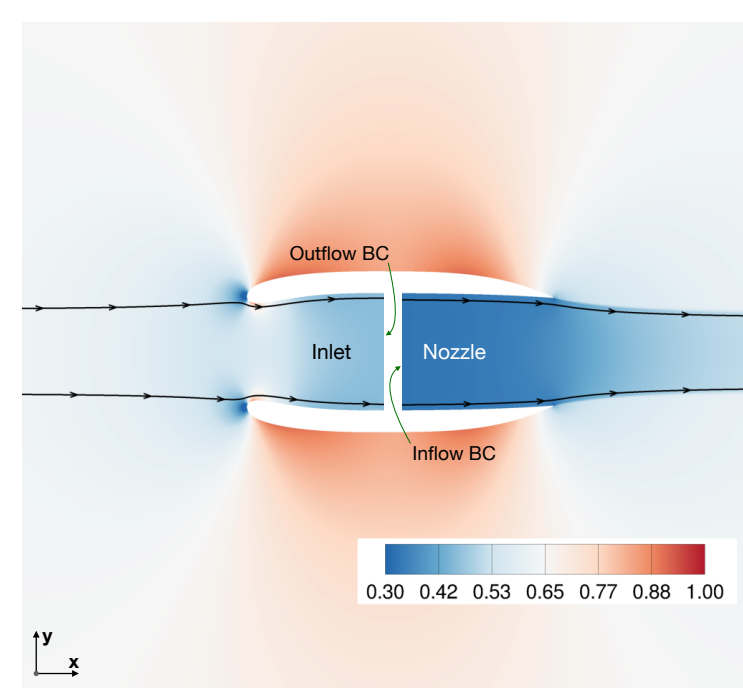

(a) Mach number contours and flow streamlines

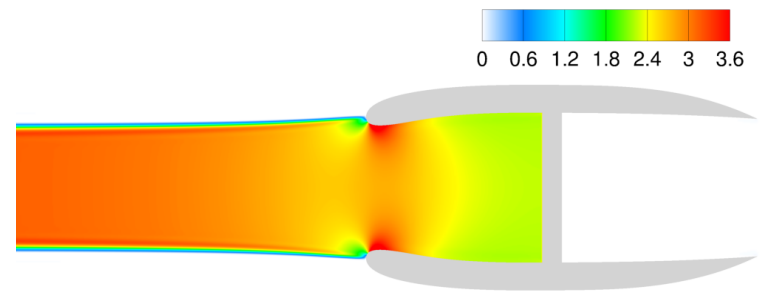

(b) $x$-momentum adjoint contours

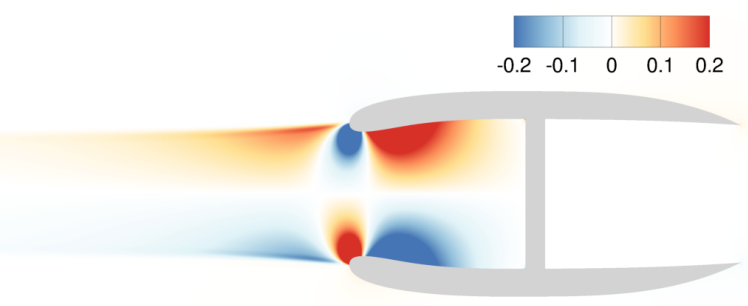

(c) $y$-momentum adjoint contours

Figure 2: Near-body flow and adjoint solutions for a simple nacelle problem $\left(M_{\infty}=0.65\right)$. Output functional is $J_{\dot{m}}$ at the outflow of the inlet and the boundary condition is specified pressure at inlet outflow.

The left-hand side of Eq. 22 is typically rank deficient. This constrains the choice and the number of flow variables that can be used in the formulation of the output functional. ${ }^{27,29}$ For example, at the slip-wall boundary, $U_{n}=0$, the transpose of the flux Jacobian $\partial(\mathbf{F} \cdot \hat{\mathbf{n}}) / \partial \mathbf{q}_{b}$ is a rank-one matrix, which constrains the output to one free parameter, specifically the output must be a function of only pressure. ${ }^{30,31}$ For the permeable boundary conditions, the flux Jacobian may be full rank, but the leading matrix, the Jacobian $\partial \mathbf{q}_{b} / \partial \mathbf{q}$, is generally rank deficient.

The boundary state for subsonic outflow with specified exit pressure is given by Eq. 10. Since $p_{\text {set }}$ is a constant, the matrix $\partial \mathbf{q}_{b} / \partial \mathbf{q}$ contains a zero row and is rank three in $2 \mathrm{D}$. Using the approach of Berg and Nordström, ${ }^{32}$ Huang et al. ${ }^{27}$ show that the pressure boundary condition is well posed, in particular as a farfield condition, and they note that it should remain well posed even with more general outputs. This should hold for $J_{\dot{m}}$, since Eq. 23 depends on three states that match the matrix rank. While the full analysis is beyond the scope of this paper, we perform a numerical study to investigate the adjoints near the wall.

Figure $2 \mathrm{a}$ shows the near-body flow around a two-dimensional, simple nacelle consisting of an inlet (diffuser) and a converging nozzle. The freestream Mach number is 0.65 . We specify $p_{\text {set }}$ at the outflow of the inlet and also measure $J_{\dot{m}}$ at this boundary. We impose a subsonic inflow condition at the entrance of the nozzle, which we discuss later. Slip-wall boundary conditions are used everywhere else on the wetted surface and the farfield is roughly ten body-lengths away. Figures $2 \mathrm{~b}$ and $2 \mathrm{c}$ show the adjoint solution for the $x$ and $y$-momentum equations, respectively. The adjoint reveals the sensitivity of $J_{\dot{m}}$ to perturbations in the local residual. The main feature of the solution is the inlet streamtube; the adjoints are small everywhere else. This means that beyond the stagnation streamlines residual perturbations have very little influence on the mass flow rate through the inlet. The $y$-momentum adjoint vanishes at the outflow boundary because perturbations tangential to the face do not affect the mass flow through the face. Most importantly, the adjoint solution is smooth near all wall boundaries, showing no spurious oscillations and indicating adjoint consistency.

Next, we examine the normal exit velocity boundary condition given by Eq. 11. This boundary condition has received much less attention in the literature and the analysis of Berg and Nordström ${ }^{32}$ is inconclusive regarding well-posedness. The matrix $\partial \mathbf{q}_{b} / \partial \mathbf{q}$ is rank deficient, but it is not clear if the free parameters of $J_{\dot{m}}$ properly align with the non-zero rows of the matrix. To investigate, we repeat the nacelle numerical study, but specify $U_{\mathrm{n}, \mathrm{set}}$ instead of $p_{\text {set }}$. Figure 3 shows a close up of the inlet portion of the flowfield for both boundary conditions plotted with discrete contours. The adjoint solutions are remarkably similar. Close examination of Fig. 3b reveals that there is some variation in the adjoint values propagating upstream from the corners of the outflow boundary, where the cut cells include both slip-wall and $U_{\mathrm{n} \text {,set }}$ boundaries. There is also an increase in the adjoint value at the outflow boundary, which is larger for the $U_{\mathrm{n} \text {,set }}$ boundary condition. 


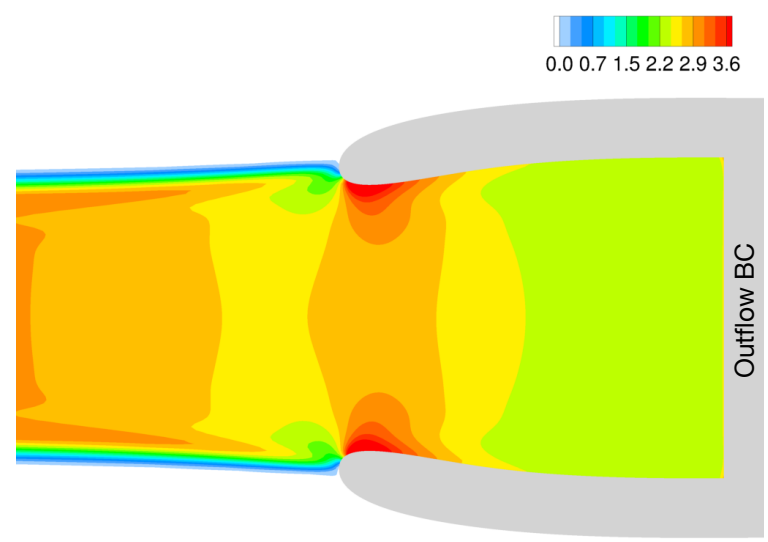

(a) Pressure BC

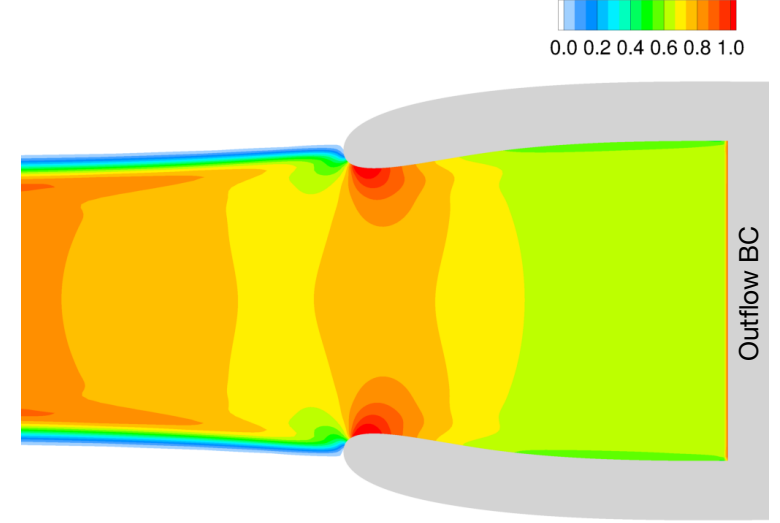

(b) Velocity BC

Figure 3: Comparison of $x$-momentum adjoint contours for the outflow pressure and velocity boundary conditions. Output functional is $J_{\dot{m}}$ at the outflow of the inlet.

Despite these variations, our experience on practical problems indicates no significant performance differences between the two outflow boundary conditions.

For the inflow boundary conditions, we first consider the case where we specify the stagnation quantities $T_{\mathrm{t}, \mathrm{set}}$ and $p_{\mathrm{t}, \mathrm{set}}$. The boundary state is given by Eq. 12 and the matrix $\partial \mathbf{q}_{b} / \partial \mathbf{q}$ is again rank deficient. We modify the nacelle test case to examine the boundary adjoint. As indicated in Fig. 2a, the numerical studies performed so far already utilize an inflow boundary condition at the nozzle entrance. The $\left[T_{\mathrm{t}, \mathrm{set}}, p_{\mathrm{t}, \mathrm{set}}\right]$ boundary condition has been used in all cases. For an inflow output, we move $J_{\dot{m}}$ from the outflow of the inlet to the inflow of the nozzle. Figures $4 \mathrm{a}$ and $4 \mathrm{~b}$ show the adjoint solution for the $x$ and $y$-momentum equations, respectively. The adjoint solution is smooth at all wall boundaries providing empirical evidence that the boundary adjoint is well posed. The notable upstream extent of the adjoint solution is expected because the interaction between the wake and the nozzle plume affects $J_{\dot{m}}$ and depends on the shape of the propulsive streamtube and the shape of the nacelle. In addition, it is interesting to compare Figs. $4 \mathrm{a}$ and $2 \mathrm{~b}$. The adjoint solutions are reciprocal in the sense that they are non-zero on the exterior or in the interior of the propulsive streamtube, depending on the location of $J_{\dot{m}}$.

For the final inflow boundary condition we specify $T_{\mathrm{t}, \text { set }}$ and $\dot{m}_{\text {set }}$. The boundary state is given by Eq. 13, where the sole interior variable is density. This implies that the left-hand side of Eq. 22 is a rank-one matrix. Since $J_{\dot{m}}$ involves three parameters in general, we expect the adjoint to be ill-posed. It is important to note, however, that measuring $J_{\dot{m}}$ at a boundary where it is being set explicitly is impractical. ${ }^{a}$ For completeness, we rerun the nacelle problem with specified $\left[T_{\mathrm{t}, \mathrm{set}}, \dot{m}_{\text {set }}\right]$. Figure 5 shows that the adjoints are zero everywhere except in the cells adjacent the inflow boundary, where the adjoint solution is oscillatory.

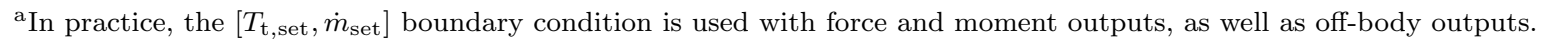




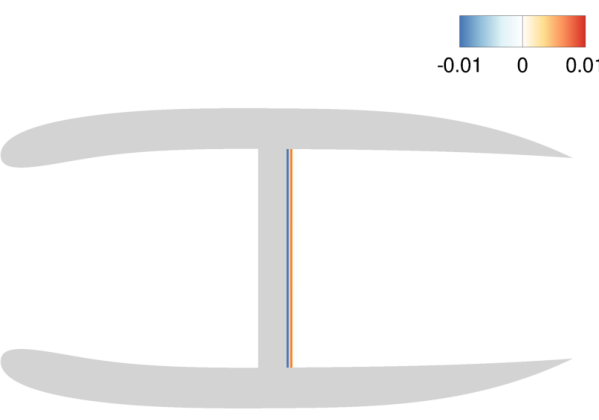

Figure 5: $x$-momentum adjoint contours for $\left[T_{\mathrm{t}, \mathrm{set}}, \dot{m}_{\text {set }}\right]$ inflow BC. Output is $J_{\dot{m}}$ at nozzle inflow.

\section{Aerodynamic Shape Optimization}

In addition to error estimation, we also use the adjoint solution for the computation of gradients in aerodynamic shape optimization. Consider determining values of design variables $\mathbf{X}$ that minimize a scalar objective function

$$
\min _{\mathbf{X}} \mathcal{J}(\mathbf{X}, \mathbf{Q})
$$

subject to constraints

$$
C_{j}(\mathbf{X}, \mathbf{Q}) \leq 0 \quad j=1, \ldots, N_{\mathrm{c}}
$$

where $\mathcal{J}$ and $C_{j}$ may involve Eqs. 3-5, e.g., drag minimization at fixed lift and fixed engine mass flow rate, and $\mathbf{Q}$ satisfies Eq. 1 for any $\mathbf{X}$ within a feasible region of the design space. The design variables $\mathbf{X}$ not only control the shape of the vehicle, but may also include $\left[p_{\text {set }}, U_{\mathrm{n}, \mathrm{set}}\right]$ and the freestream parameters $\left[M_{\infty}, \alpha, \beta\right]$.

We use the numerical method and error estimates outlined in Secs. III-V in conjunction with the SNOPT numerical optimization software ${ }^{33}$ to solve Eqs. 24-25. Since the error estimation and mesh adaptation procedure is integrated within the optimization, we are able to terminate the optimization when the design improvements are within a prescribed level of discretization error. ${ }^{16}$ Put another way, we minimize overoptimization for a given level of accuracy (mesh refinement). Details of the methodology, including the evaluation of gradients, are available in earlier work. ${ }^{34,35}$ Here we focus on the gradient $\mathrm{d} J_{H} / \mathrm{d} \mathbf{X}_{i}$ when

$\mathbf{X}=\left[p_{\mathrm{set}}, U_{\mathrm{n}, \mathrm{set}}\right]^{\mathrm{T}}$. We rewrite Eq. 6 to explicitly include the design variables, resulting in a system of equations

$$
\mathbf{R}_{H}\left(\mathbf{X}, \mathbf{Q}_{H}\right)=0
$$

The gradient is obtained by linearizing the objective function and the residual, resulting in the following expression

$$
\frac{\mathrm{d} J}{\mathrm{~d} \mathbf{X}_{i}}=\frac{\partial J}{\partial \mathbf{X}_{i}}-\boldsymbol{\psi}^{\mathrm{T}} \frac{\partial \mathbf{R}}{\partial \mathbf{X}_{i}}
$$

where the adjoint vector $\boldsymbol{\psi}$ is given by Eq. 18 and we omit the subscript $H$ since all gradient computations are performed on the working mesh. Equation 27 can be simplified further because Eqs. 3-5 do not explicitly depend on $p_{\text {set }}$ or $U_{\mathrm{n} \text {,set }}$ and therefore $\partial J / \partial \mathbf{X}_{i}=0$. When $\mathbf{X}$ controls the shape of the vehicle, e.g., via spline control points, the expression for the gradient is more complicated because the influence of shape design variables is implicit via the mesh and the triangulation of the wetted surface. For details see Ref. 34 .

\section{Results}

\section{A. Code Verification: Mass-Flow-Rate in a Two-Shock Wedge}

We begin with a two-dimensional test with an exact solution that mimics a supersonic inlet. ${ }^{24}$ As illustrated in Fig. 6a, supersonic flow is decelerated through a system of two oblique shocks using a simple wedge geometry. Our goal is to compute the mass-flow-rate at the outflow boundary inside the duct. The analytic mass flow rate is independent of the flow state inside the inlet if there is no spillage. This is because the area of the inlet streamtube is fixed by the capture area, $A_{c}$ in Fig. 6a. Hence, $\mathcal{J}_{\dot{m}}=\rho_{\infty} u_{\infty} A_{c}$, which equals -0.4138 


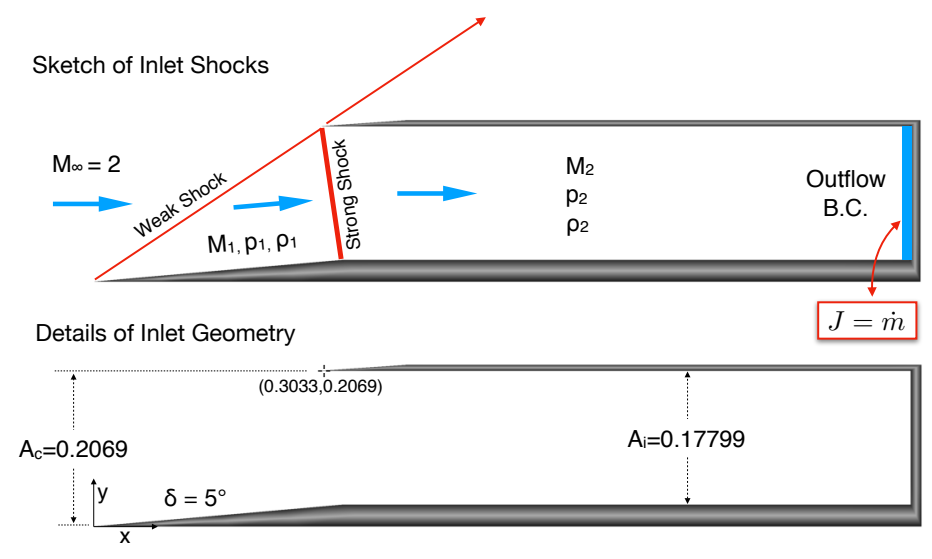

(a) Inlet geometry and sketch of flowfield

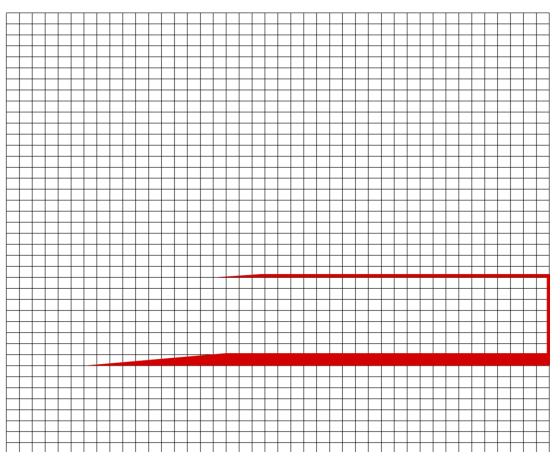

(b) Initial mesh: 1,654 cells

Figure 6: Two-shock wedge diffuser: problem setup and computational domain

in nondimensional form ${ }^{\mathrm{b}}$. Uniform mesh refinement is performed to determine the formal order of accuracy of $J_{\dot{m}}$ and the sharpness of the error estimate. This is followed by a mesh adaptation test.

We use the exit-pressure boundary condition, Eq. 10, with $p_{\text {set }}=4.850557 p_{\infty}$. This value is determined analytically through use of the oblique shock relations to calculate the flow conditions behind the first (weak) shock and then the second (strong) shock. By design, the weak shock impinges perfectly on the upper lip. In other words, there is no spill and the strong shock turns the flow parallel to the inlet walls without an expansion at the lower surface corner. ${ }^{24}$ For $M_{\infty}=2$ and wedge deflection of $5^{\circ}$, the static pressure jump across the weak shock is approximately 1.3154 , and 3.6875 across the strong shock.

The uniform mesh refinement study involves a sequence of six nested meshes. Figure $6 \mathrm{~b}$ shows the computational domain and the initial mesh. Farfield boundaries are used everywhere except at the inlet's outflow and the slip-walls. The initial mesh contains 1,654 cells with cell-size $H \approx 0.025$. The finest mesh contains 1,677, 895 cells. Note that the boundary discretization changes non-smoothly with refinement. Consequently, error convergence rates are obtained via linear regression of the finest four solutions. The spatial discretization uses the minmod limiter and convergence to steady-state is accelerated via three-level multigrid.

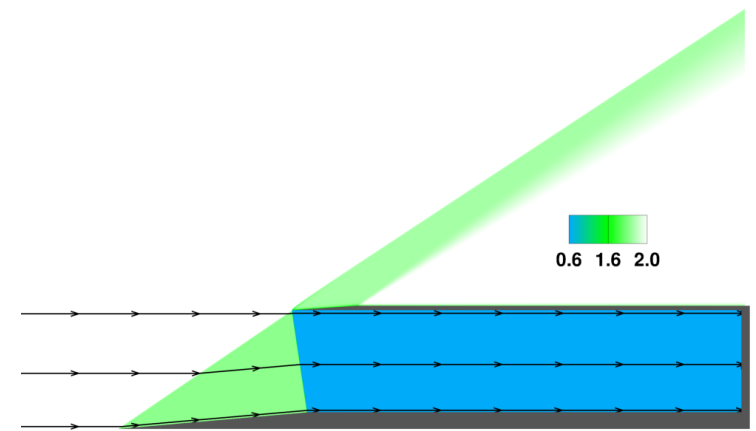

(a) Mach number and flow streamlines

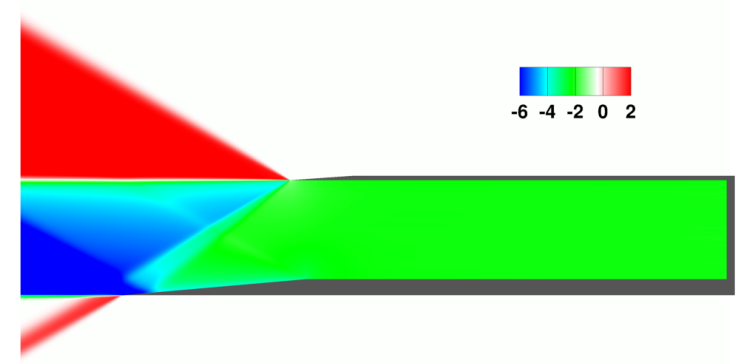

(b) Adjoint for the mass conservation equation and $J_{\dot{m}}$ functional

Figure 7: Flow and adjoint solutions for the two-shock wedge on the finest (1.7M cells) uniform mesh

Before investigating functional accuracy, we discuss the salient features of the flow and adjoint solutions. Figure $7 \mathrm{a}$ shows the Mach number contours and flow streamlines. The solution converges to the expected two-shock system and the streamlines indicate that there is no spillage around the upper

\footnotetext{
${ }^{\mathrm{b}}$ The negative sign indicates that the flow is leaving the computational domain, $\rho_{\infty}=1$ and $u_{\infty}=M_{\infty}=2$
} 
lip. Figure $7 \mathrm{~b}$ shows the solution for the density adjoint. The contours show the sensitivity of $J_{\dot{m}}$ to local perturbations in mass conservation. The adjoint solution is continuous across the shocks and changes sign across the boundaries of the inlet streamtube. Furthermore, the adjoint values are zero outside the functional's zone of dependence, i.e., downstream of the characteristics impinging on the upper and lower lips. Inside the inlet, the adjoint values are moderate and essentially uniform, with no oscillations or discontinuities near the inlet's outflow boundary. This agrees well with the analysis and numerical experiment presented in Section V (Fig. 2), and provides further empirical evidence of adjoint consistency.

Turning to output accuracy, Fig. 8 shows that the error in $J_{\dot{m}}$ is $\mathcal{O}(h)$. The first line, labeled Exact, is the error in $J_{\dot{m}}$ as measured with respect to the analytic solution. First-order accuracy is expected since the flow is dominated by shocks. The second line, labeled Estimate, is the adjoint estimate of the discretization error, Eqs. 16 and 21 . The estimate is only slightly below the true error. Its sharpness improves over the first four meshes, but fades on the finest mesh. The discrepancy may be due to the presence of shocks in the flow solution and discontinuities in the adjoint solution, which may cause inaccuracies in

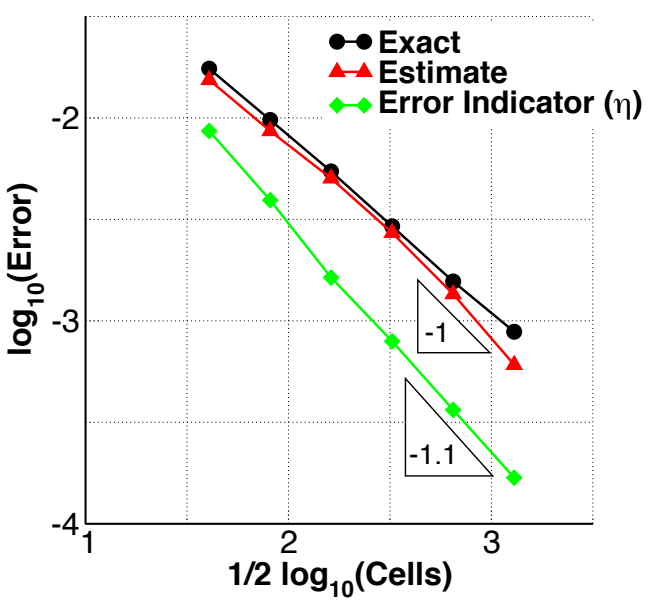

Figure 8: $J_{\dot{m}}$ error convergence with uniform mesh refinement the reconstruction procedures to the embedded mesh. This is in addition to the non-smooth changes in the cut-cell geometry with refinement. The third line in Fig. 8, labeled Error Indicator, is $\eta=\mathrm{L}_{1}|\boldsymbol{\eta}|_{H}$. Recall $\boldsymbol{\eta}_{H}$ is the error indicator that drives mesh adaption via Eq. 20. This is a higher-order term. We observe that its value is significantly smaller than the true error and it is vanishing at a slightly higher rate.

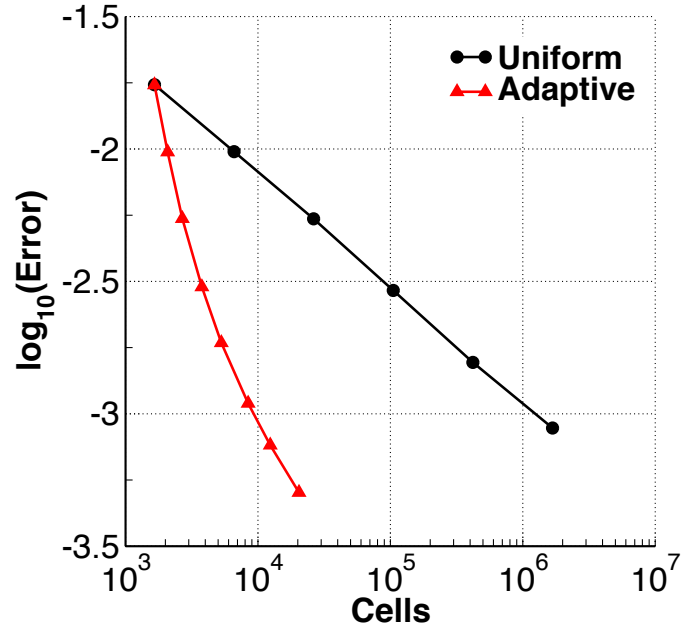

(a) Comparison of convergence of error in $J_{\dot{m}}$ using uniform and adaptive refinement

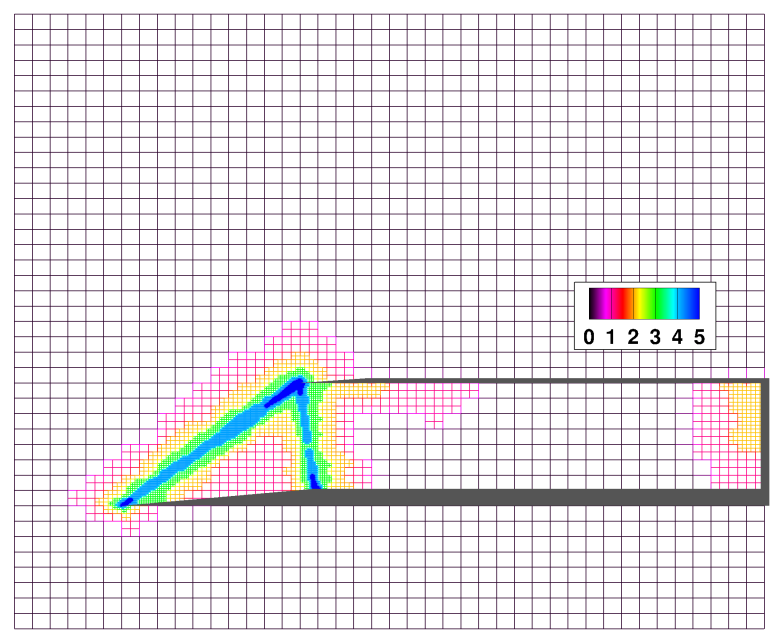

(b) Mesh after 5 adaptation cycles (8,427 cells). Color represents cell refinement level, where level 0 denotes cells of the initial mesh.

Figure 9: Adaptive mesh refinement of the two-shock wedge for $J_{\dot{m}}$.

Having established the accuracy of $E$ and $\eta$, we examine their usefulness in adaptive mesh refinement. We perform seven adaptive refinements starting from the initial mesh shown in Fig. 6b. The adaptation threshold ${ }^{\mathrm{C}}$ is set to be a factor of two smaller than the mean error value in each adaptation cycle $\left(\eta / N_{c} / 2\right)$ based on benchmark results from Ref. 15. Figure 9a shows the error convergence of $J_{\dot{m}}$ with comparison to the uniform refinement study. We observe that after each refinement the error nearly matches the value obtained on the uniformly refined mesh, but uses far fewer cells. For example, after the fifth adaptation the

${ }^{\mathrm{c}}$ Cell $i$ in the working mesh is refined if $\boldsymbol{\eta}_{i}$ exceeds the threshold value. 


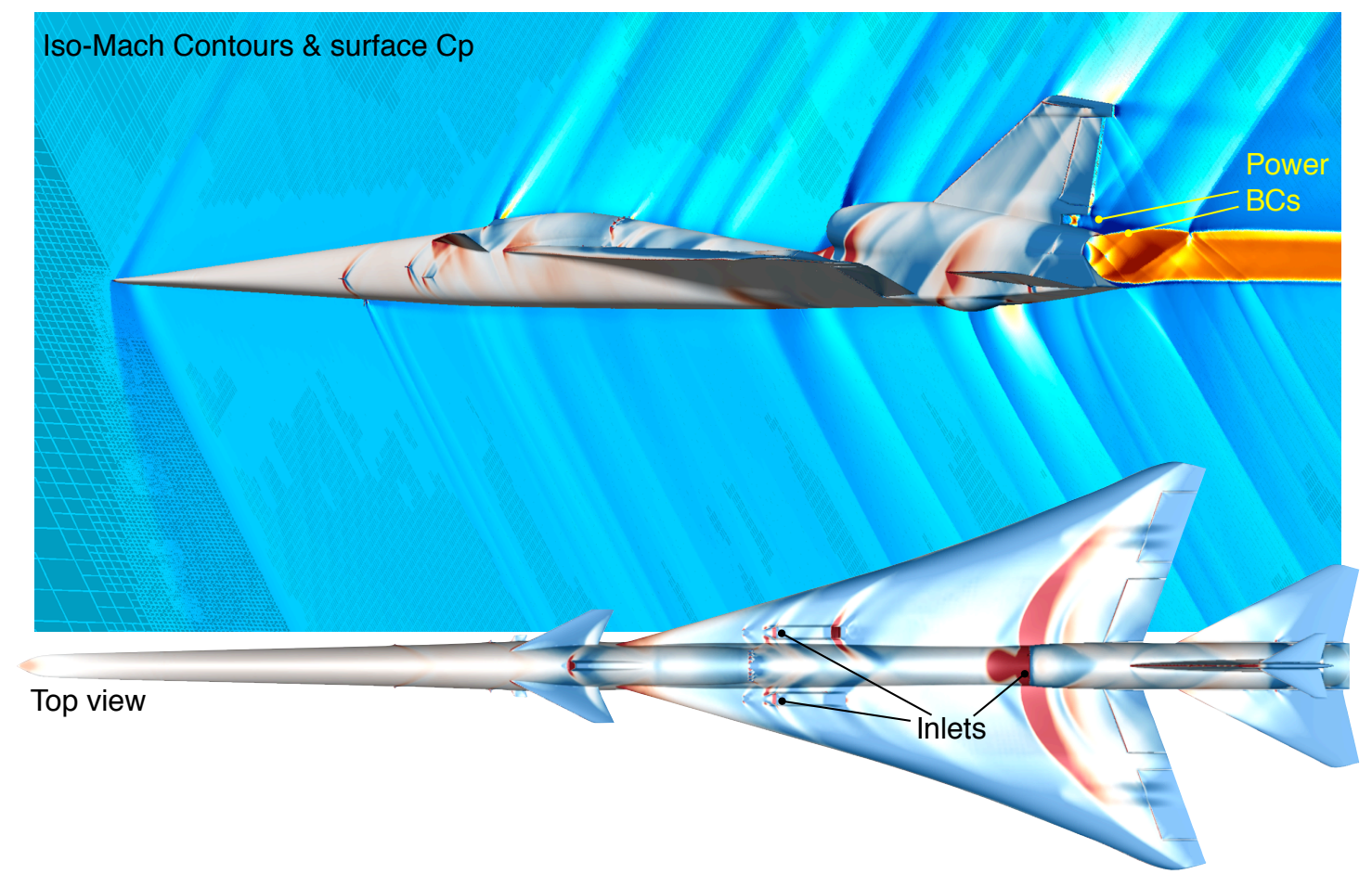

Figure 10: Low-boom aircraft configuration at $M_{\infty}=1.4$ and $\alpha=2.05^{\circ}$ with control surfaces set for level flight at $54 \mathrm{kft}$. The mesh is shaded with iso-Mach contours while the aircraft surface is colored by $C_{p}$.

mesh contains only 8,427 cells compared to 1,677,895 cells on the uniformly refined mesh, with very similar error levels. After the sixth refinement cycle, the rate of convergence of the adaptation starts to approach the uniform refinement. This suggests that the error is reaching equidistribution among the cells and we note a corresponding increase in the mesh growth.

Figure 9b shows the adapted mesh after the fifth refinement cycle. The finest cells track the inlet shocks. There is no refinement outside the zone of dependence of the output. For example, the shock generated by the upper wedge is ignored because the adjoint values are zero in this region, as shown in Fig. 7b. Inside the inlet, there is essentially no refinement except for the outflow boundary where $J_{\dot{m}}$ is defined. This is consistent with the uniform flow and adjoint solution inside the inlet.

\section{B. Low-Boom Aircraft Analysis}

\section{Mass-Flow-Rate Outputs}

This example represents a typical analysis case for a low sonic-boom aircraft. We compare simulations with and without $J_{\dot{m}}$ outputs to assess the usefulness of these outputs in practical simulations. Figure 10 shows an overview of a highly detailed model of NASA's X-59 low-boom flight demonstrator, as well as the mesh and flow solution on the symmetry plane. This particular configuration corresponds to a near final design and includes not only the airframe, control surfaces, engine inlet and nozzle, but also inlets and vents for the Environmental Control System (ECS), engine bay vents and other geometric detail. There are three inlets, where flow enters the vehicle. These are shown in the top view in Fig. 10, with labels pointing to the forward ECS inlets and vent, and the engine inlet. We use the exit pressure outflow boundary condition, Eq. 10, for all inlets. There are also four inflow boundary conditions, where high-temperature flow exits the vehicle and enters the computational domain, labeled as "Power BCs" in Fig. 10 . Here we specify $T_{\mathrm{t} \text {,set }}$ and $p_{\mathrm{t} \text {,set }}$ with the boundary state given by Eq. 12 . The simulation is performed at cruise conditions: $M_{\infty}=1.4, \alpha=2.05^{\circ}$ and altitude of $54,000 \mathrm{ft}$.

The central challenge in performing simulations for boom prediction is the necessity to accurately predict very small pressure fluctuations many aircraft lengths below the vehicle. ${ }^{\mathrm{d}}$ We use off-body line sensors $J_{l}$, given

\footnotetext{
${ }^{\mathrm{d}}$ In practice this means predicting pressure fluctuations of less than a Pascal at a distance of over $100 \mathrm{~m}$ from the vehicle.
} 


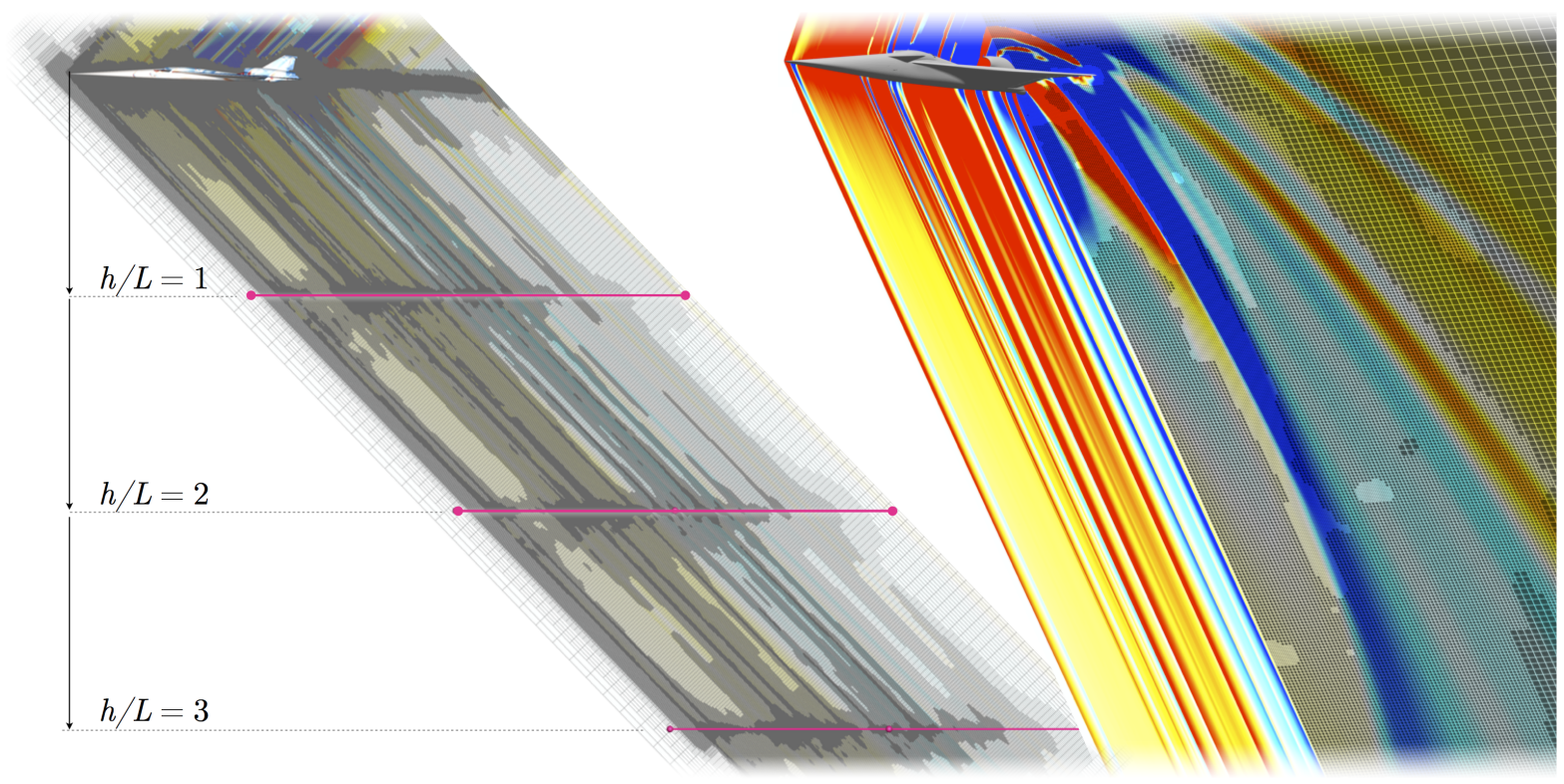

Figure 11: Left: Adapted mesh and sensor locations in a plane located on the aircraft's centerline. Right: Front three-quarter zoom with $C_{p}$ contours in the symmetry plane and a slice of the mesh on a crossflow plane swept at the Mach angle.

by Eq. 5 with $n=2$ and $\omega=1$, as the primary outputs of interest. We have shown in prior work ${ }^{36,37}$ that these outputs result in efficient meshing for boom-prediction problems. Figure 11 shows a typical adapted mesh for the low-boom configuration in Fig. 10. The aircraft reference length for boom is $L_{\text {Ref }}=90$ feet and pressure signatures are being extracted along sensors at distances of 90,180 and 270 feet below the flight path $\left(h / L_{\text {Ref }}=\right.$ $\{1,2,3\})$. The net adaptation functional is a linear combination of $J_{l}$ outputs with a weighting proportional to $h / L_{\text {Ref }}$. The mesh shown in Fig. 11 has $\sim 144 \mathrm{M}$ cells after 8 adaptation cycles and clearly shows the meshing needed to minimize error in the propagation to the sensor locations. The image on the left shows the mesh and sensor locations in a plane located on the aircraft's centerline. On the right, a zoomed-in three-quarter view shows $C_{p}$ contours on the symmetry plane and a slice on a crossflow plane, which is swept at the Mach angle. Figure 12 shows the convergence history of the adaptation functional. Beyond $10^{7}$ cells, the functional begins converge and the error estimates are small.

While $J_{l}$ is an effective way to rapidly obtain reliable off-body pressure signatures, it only guarantees accurate mass flow rates through the permeable boundaries in the high-resolution limit of mesh refinement. Put another way, the mass flow rates through the inlets, vents and nozzles are likely slow to converge, potentially leading to inaccurate prediction of aerodynamic performance. To investigate this effect, we include $J_{\dot{m}}$ for each permeable boundary condition in the adaptation functional. Since $J_{\dot{m}}$ scales linearly with the areas of the various inlets and nozzles, we choose to weight these with a constant proportional to the inverse of their flow-through area. The inverse-area weighting is optional, but it ensures that small inlets begin attracting refinement earlier in the adaptive process and roughly balance the $J_{\dot{m}}$ sensors on all the permeable boundaries.

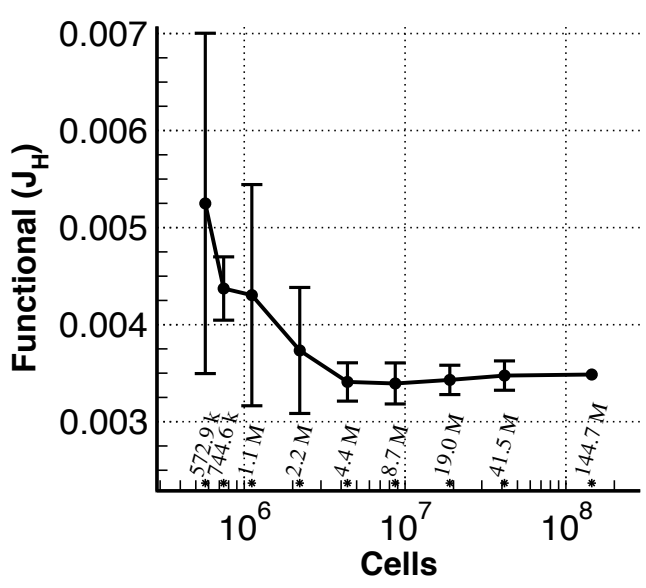

Figure 12: Adaptation functional convergence history with error bars showing level of discretization error.

The left plot of Fig. 13 shows the off-body pressure signature at $h / L=3$ directly under the flight path. The figure compares two simulations: with and without $J_{\dot{m}}$ labeled as "MFR" and "No MFR", respectively, added to the standard $J_{l}$ outputs. While these near-field 

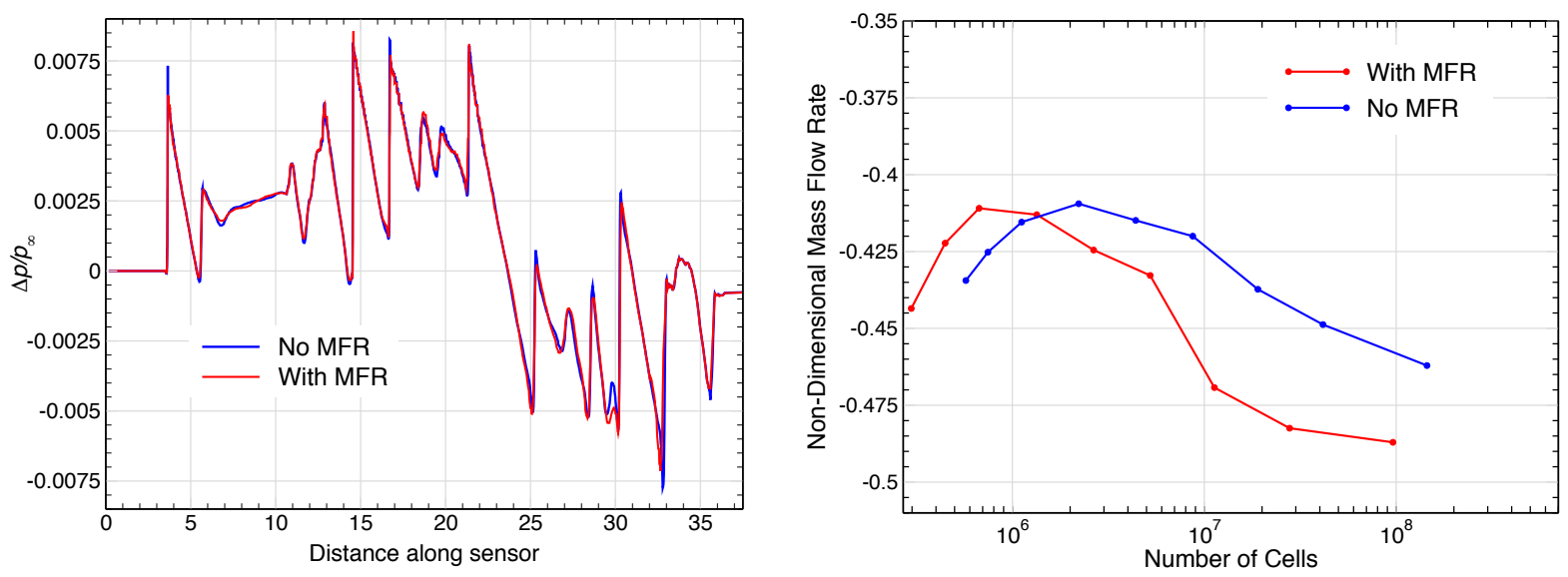

Figure 13: Left: Near field pressure signature at $h / L=3$ under-track for cases with and without mass flux in the objective. Right: Mesh convergence of $J_{\dot{m}}$ through the main inlet.

pressure signatures are essentially identical, it is interesting to examine the convergence of mass flow rate through the various permeable boundaries. The plot on the right of Fig. 13 examines $J_{\dot{m}}$ convergence for the main engine inlet. It is clear that the mass flow rate for the "No MFR" case is not mesh converged. Considering that the finest mesh without $J_{\dot{m}}$ outputs is quite large $(\sim 144 \mathrm{M}$ cells), this is initially somewhat surprising. Convergence of mass flow rates is much better with $J_{\dot{m}}$ added. The mass flow rate is converged to within $4 \%$ on a mesh with over an order of magnitude fewer cells.
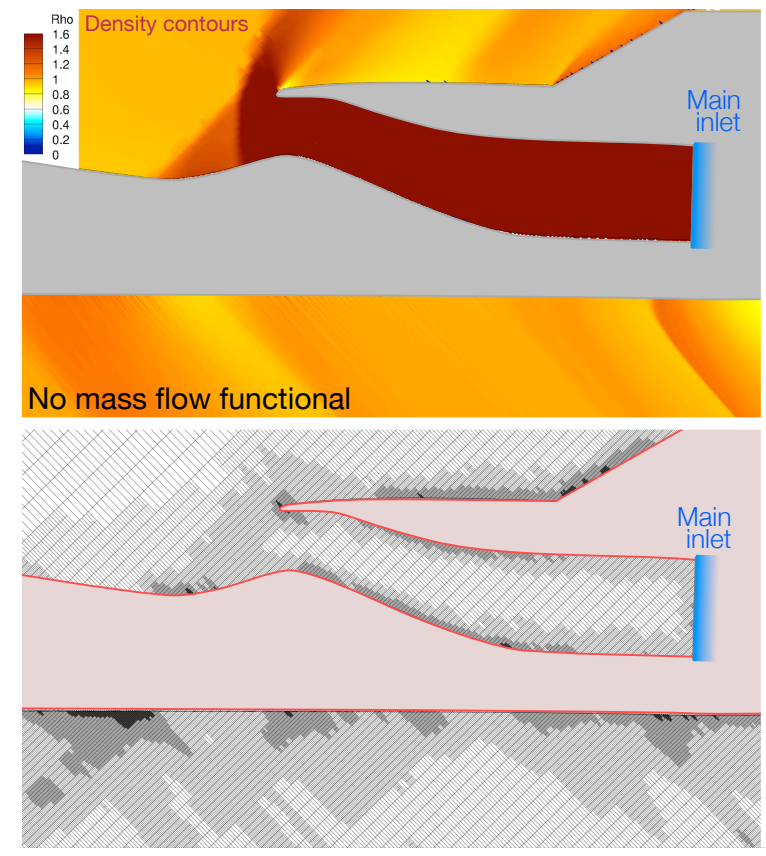
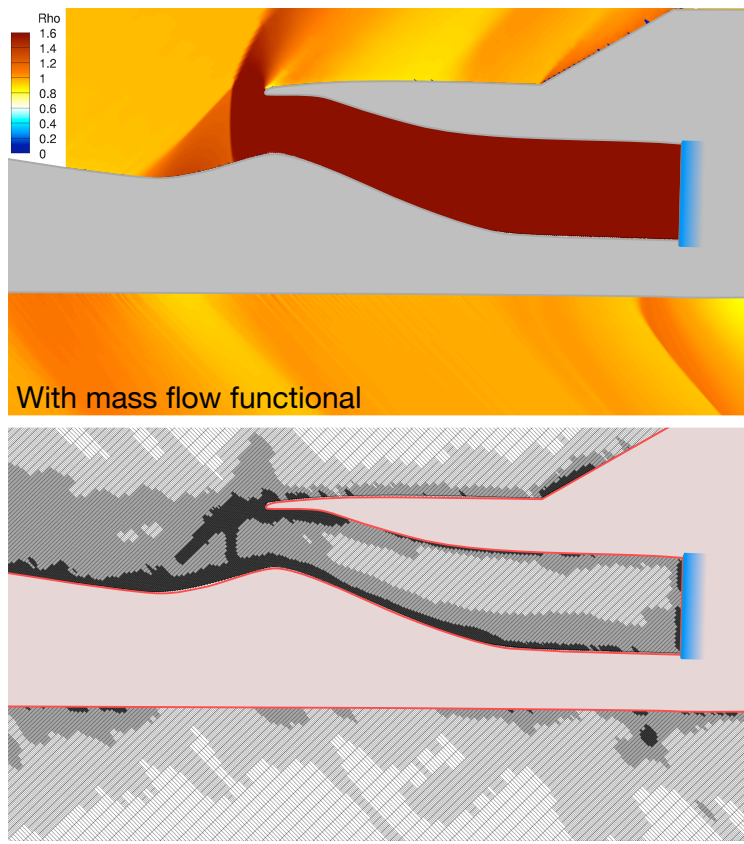

Figure 14: Contours of density and view of the mesh down the centerline of the main inlet Left: Standard setup without $J_{\dot{m}}$ in adaptation functional Right: With $J_{\dot{m}}$

To understand how the addition of $J_{\dot{m}}$ influences the mesh, consider the snapshots in Fig. 14 . On the left is the standard setup, with only $J_{l}$ outputs, while the images on the right show the solution and mesh with the addition of $J_{\dot{m}}$. The upper two images show density contours of the solution, while the lower two present views of the mesh through the centerline of the inlet. When $J_{\dot{m}}$ is included, the density contours show much better resolution of the cowl-lip shock structure, and it is clear from the mesh that these structures are being meshed more aggressively. Moreover, the resolution of the entire capture streamtube of the main inlet is 
improved, and the finer cells extend far upstream. The mesh within the S-duct is also significantly finer to reduce errors in the flow leading up to the outflow (compressor face) boundary.

\section{Mass-Flow-Rate Control}

Building on the analysis case above, we consider a simple optimization problem to achieve target mass flow rates through specified outflow boundaries. The purpose is to demonstrate gradient accuracy when design variables involve the boundary states, i.e., Eq. 27. Using the same aircraft geometry and conditions shown in Fig. 10, we prescribe target mass flow rates for the main engine intake and the two ECS intakes on the upper surfaces of the left and right wings. Since the analysis case used the $p_{\text {set }}$ outflow boundary condition, we select $\mathbf{X}=\left[p_{\text {set,Main }}, p_{\mathrm{set}, \mathrm{L}}, p_{\mathrm{set}, \mathrm{R}}\right]$. For a fast design turnaround, we reduce the number of adaptation cycles to five and obtain a mesh that contains $\sim 7 \mathrm{M}$ cells. The adaptation functional involves drag and $J_{\dot{m}}$. The mass flow rate through the main inlet is approximately -0.44 , which is similar to Fig. 13 . The mass flows are -0.01 in the secondary intakes. ${ }^{\text {e }}$ To demonstrate the optimization, we set a target value of -0.4 for the main inlet and target values of -0.012 for the secondary intakes. In a real application, these inputs would be provided by a propulsion specialist.

Figure 15 summarizes the results of the optimization. Since the largest deviation from the target value is on the main inlet, the optimization adjusts the back pressure associated with this outflow in the early iterations, driving the mass flow rate to essentially -0.4 in 7 design iterations as shown in Figure 15a. The mass flow rate through the secondary inlets is matched in later iterations. Figure 15b shows the convergence of the objective function and gradient, and confirms the optimal solution.

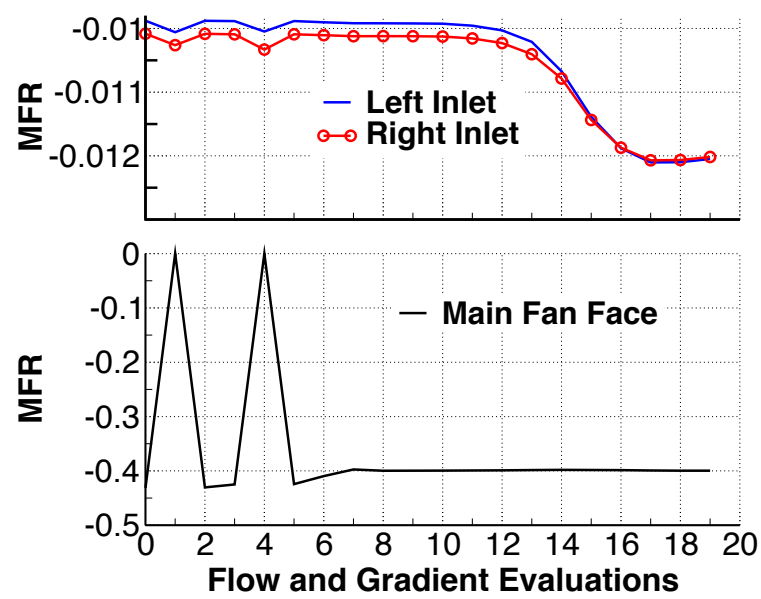

(a) Convergence of mass flow rates (MFR)

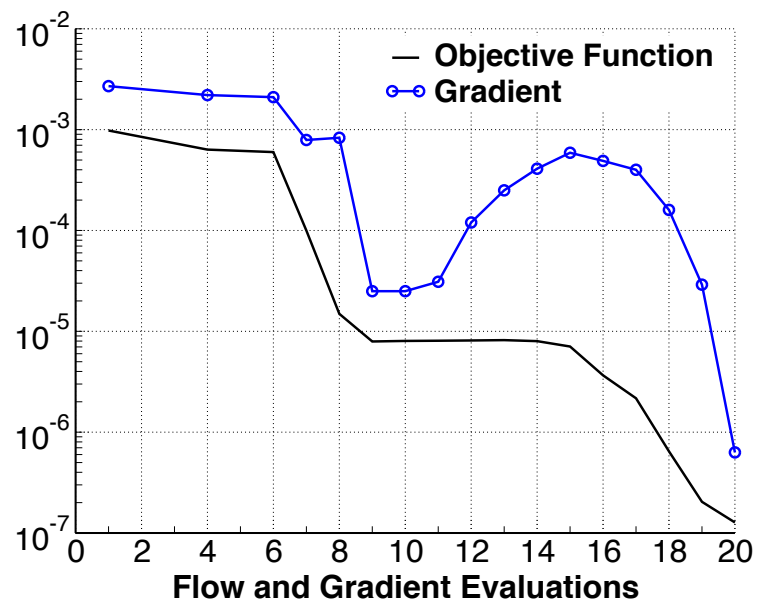

(b) Optimization convergence history

Figure 15: Optimization convergence history for the mass flow control example.

\section{Supersonic Nozzle Shape Optimization}

We consider a notional shrouded supersonic nozzle design to demonstrate shape optimization with mass-flowrate constraints and multiple inflow boundaries. This case is roughly based on the high-flow bypass nacelle design presented by Conners et al. ${ }^{1}$ and studied by Heath et al. ${ }^{38}$ to minimize nacelle effects on sonic-boom noise. Figure 16 shows the baseline geometry, which consists of a traditional axisymmetric spike nozzle that is mounted inside a shroud. The shroud acts as the outer surface of the nacelle and fully contains the inner nozzle. The duct between the nozzle and the shroud is for bypass air that can be used to attenuate flowfield pressure fluctuations generated by the core flow and thus reduce sonic-boom levels. The red surface in Fig. 16 represents the core's high-temperature inflow boundary, while the orange surface is the bypass inflow boundary. There is a subtle constriction of the bypass duct that acts as a throat. This allows for subsonic inflow at the boundary, but supersonic flow out of the bypass duct.

\footnotetext{
eThe negative sign denotes that the flow is out of the computational domain.
} 

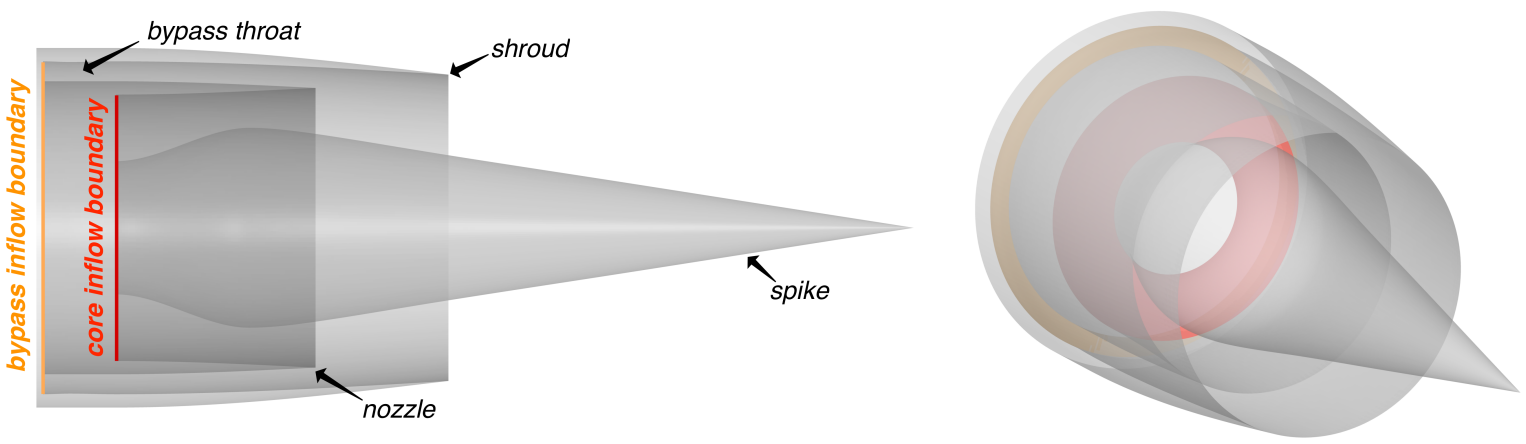

Figure 16: Two views of the shrouded spike nozzle.

Figure 17 summarizes the design variables. The spike shape, also referred to as a nozzle plug, is controlled by five radii shown in red. The spike radius is lofted streamwise with a cubic spline (black curve labeled "spike spline" in Fig. 17) with the five radial parameters providing the knots of the spline. The spike geometry from the core inflow plane to the first parameterized radius is defined by a second cubic spline ("throat spline" in Fig. 17) with fixed slopes at both ends and fixed radius at the inflow. These two spike profile splines match in slope where they meet at the first parametrized spike radius. The length of the spike is arbitrarily fixed to prevent "infinite-length" designs since the effects of weight and viscosity are ignored. The inner nozzle and the shroud walls are also controlled by cubic splines (also shown as black curves in Fig. 17). Both splines are fixed on the left ends in Fig. 17 and allowed to move radially on the right ends, thus controlling the nozzle and shroud lip radii. In total, this parameterization results in seven design variables.

Our goal is to explore the trade-off between generating maximum thrust and minimizing shocks that pollute nearfield pressure signatures. We formulate three optimization problems, which are all combinations of thrust, $J_{a}$ (Eq. 4), and a nearfield pressure signature, $J_{l}$ (Eq. 5 with $n=2$ and $\omega=1$ ), that is extracted approximately one nozzle radius below the shroud. We specify a minimum bound on each spike radius to avoid surface crossover. We use the $\left[T_{\mathrm{t}, \mathrm{set}}, p_{\mathrm{t}, \mathrm{set}}\right]$ inflow boundary condition for both the core and bypass flow. The boundary values are based on a core pressure ratio $\left(p_{\mathrm{t}, \infty} / p_{\infty}\right)$ of 6.4 , bypass pressure ratio of 3.5 , core temperature ratio $\left(T_{\mathrm{t}, \infty} / T_{\infty}\right)$ of 2.5 and bypass temperature ratio of 1.5 . Equation 3 is used to specify two mass-flow-rate constraints, one for the core and one for the bypass. The freestream Mach number is set to 1.4. Figure 18a shows the computational domain, sensor location and initial mesh. We use eight cycles of adaptive mesh refinement in each design iteration. The functional that drives the mesh refinement is a weighted sum of $J_{a}, J_{l}$ and $J_{\dot{m}}$, and consequently only a single adjoint solution is required during the adaptive process. On the finest mesh, however, three adjoints are solved: one for the objective function, which combines thrust and the pressure signature, and one for each explicit $J_{\dot{m}}$ constraint.

For the first design problem, the objective is to simply maximize thrust to establish a performance benchmark. Figure 19 shows the optimization history. Note that the constraints are satisfied and the

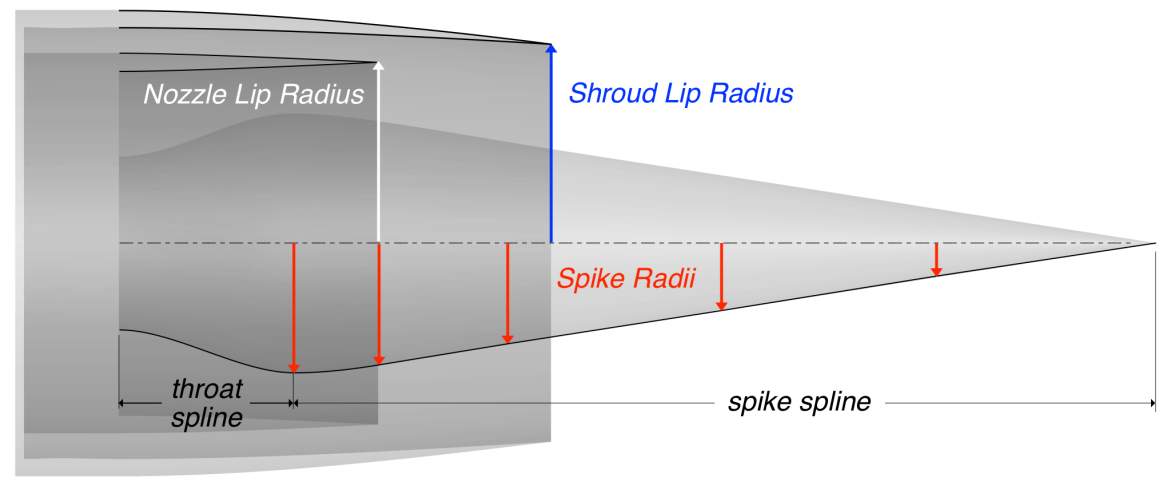

Figure 17: Spike nozzle design variables. The shroud and inner nozzle area distributions, and spike shape are controlled by cubic splines (shown as black solid curves). 


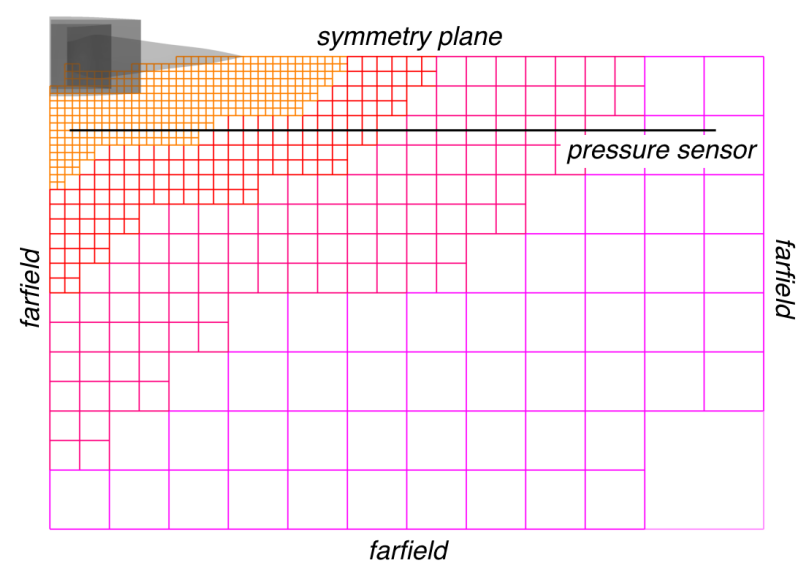

(a) Computational domain and initial mesh (5, 455 cells)

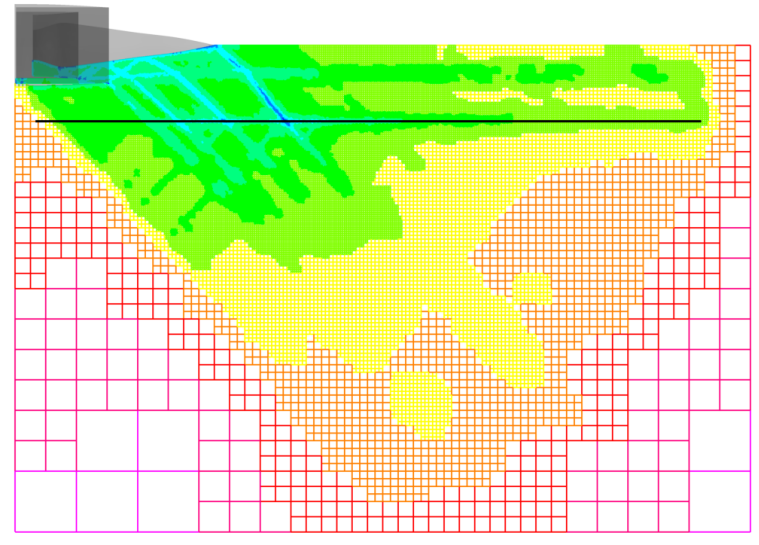

(b) Final mesh after eight adaptations ( $\sim 20 \mathrm{M}$ cells)

Figure 18: Shrouded spike nozzle problem setup. Mesh colored by refinement level.

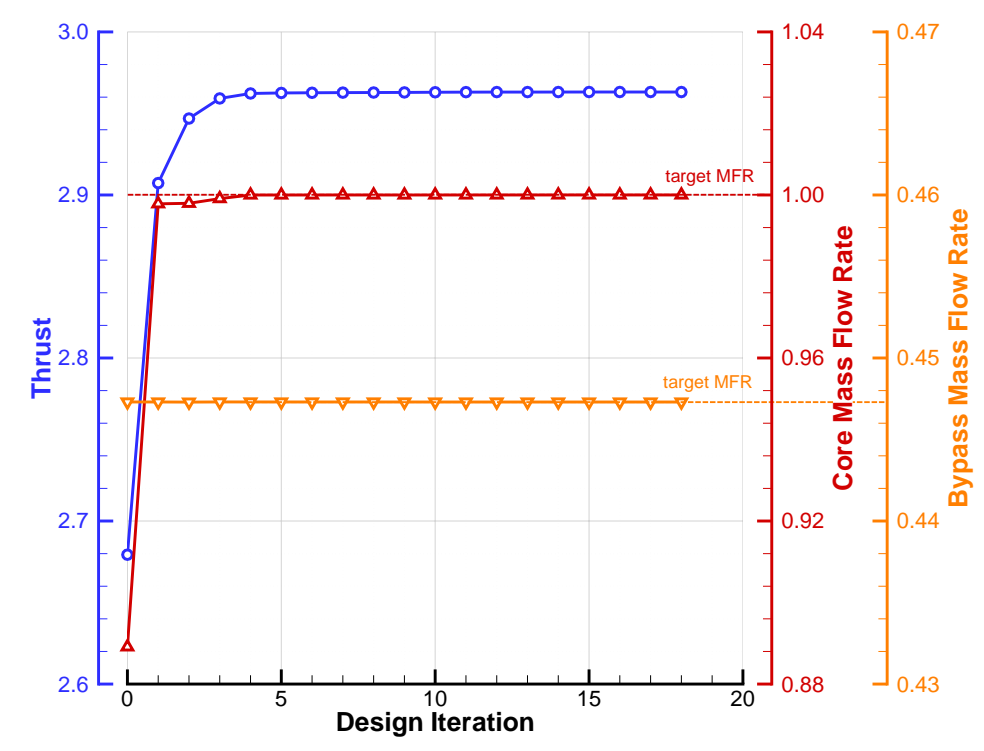

Figure 19: Convergence of objective function (thrust) and constraints (core and bypass mass flow rates) in optimization of the shrouded spike nozzle.

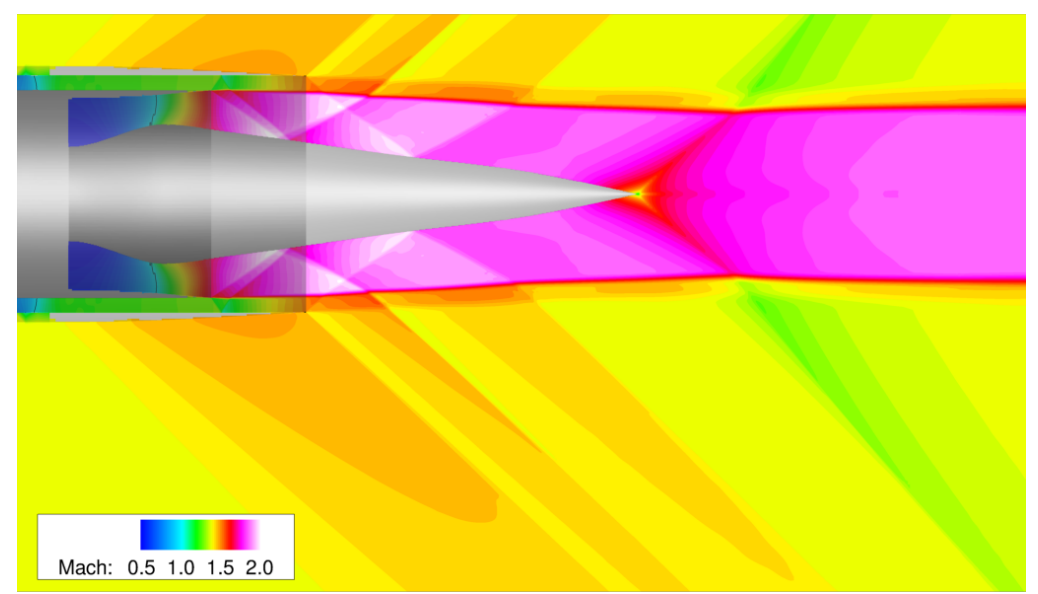

Figure 20: Mach contours of solution on centerline plane of shrouded nozzle optimized for maximum thrust. 


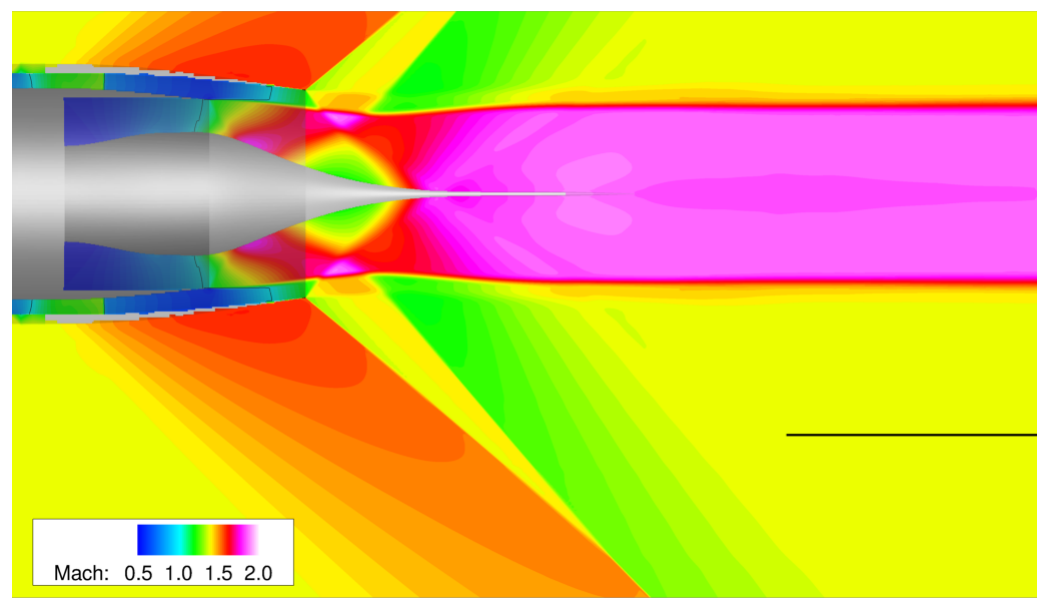

Figure 21: Mach contours of solution on centerline plane of shrouded nozzle optimized for weighted sum of maximum thrust and minimum pressure disturbance from the spike tip.

maximum thrust value is attained to plotting accuracy in about five design iterations. For this case, the SNOPT optimality metric is reduced by three orders of magnitude in 18 design iterations. While thrust has been maximized, Figure 20 shows that, as expected, the optimized shape generates strong shocks that are likely to adversely affect low-boom performance. The Mach number contours in Fig. 20 indicate sharp resolution of both the bypass flow and the complex shock structures in the core stream. The shocks originate at the nozzle and shroud lips and reflect from the nozzle spike as they propagate downstream. There is some attenuation of the shocks as they propagate through the bypass stream, particularly noticeable for the terminating conical shock at the spike tip.

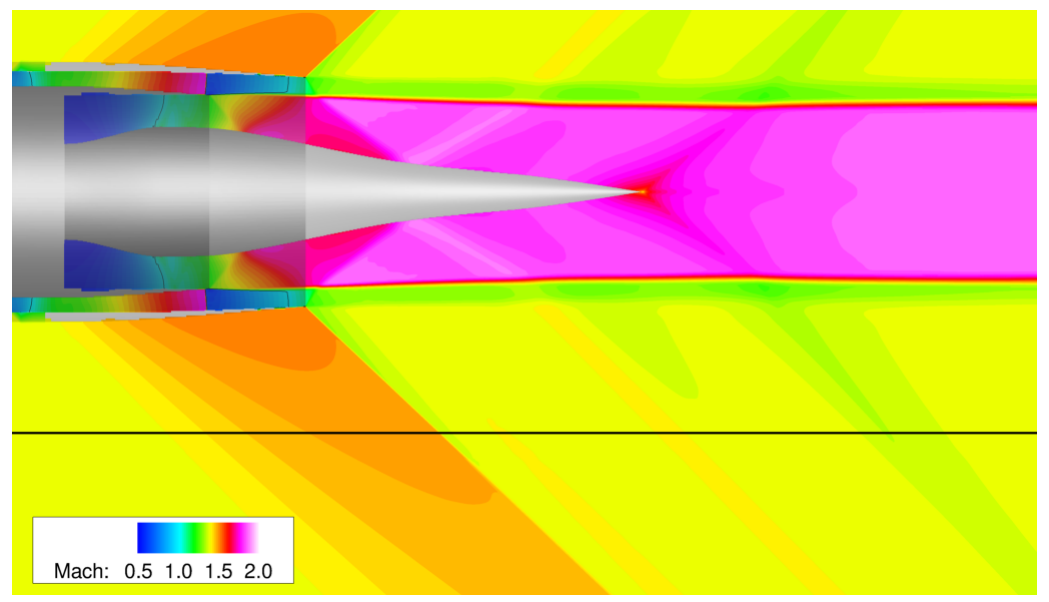

Figure 22: Mach contours of solution on centerline plane of shrouded nozzle optimized for weighted sum of maximum thrust and minimum pressure disturbance from entire nozzle.

For the second design problem, we focus on attenuating the strong conical shock at the spike tip. The primary purpose of the bypass flow and the shroud that encloses the nozzle is to help mitigate the nozzle shock. Referring to Fig. 20, it is likely that the pressure waves that emanate from the shroud lip region can be shielded by the airframe, ${ }^{2}$ and thus contribute very little to the ground pressure signature. The trailing conical shock, however, cannot be easily shielded and is likely to propagate to the ground. Consequently, the optimization involves maximizing thrust while minimizing the overpressure of the conical shock only. The weights used in the objective function are selected so that the contributions from the thrust and the pressure signature functions are roughly equal. Figure 21 reveals a dramatic design change and the Mach contours show a very different flowfield compared to the thrust maximization case. The black line in Figure 21 shows the pressure sensor. The new spike generates a gentle compression toward the spike tip and the rear conical shock is virtually eliminated. Only the minimum-bound geometric constraints prevent the spike from 
disappearing completely. This spike geometry is very similar to the design presented by Conners et al. ${ }^{1}$ Thrust is reduced by $2.7 \%$ compared to the benchmark case. Furthermore, the shocks emanating from the nozzle lip region are stronger than those in the first design problem, perhaps so much so that they could affect the ground pressure signature despite airframe shielding.

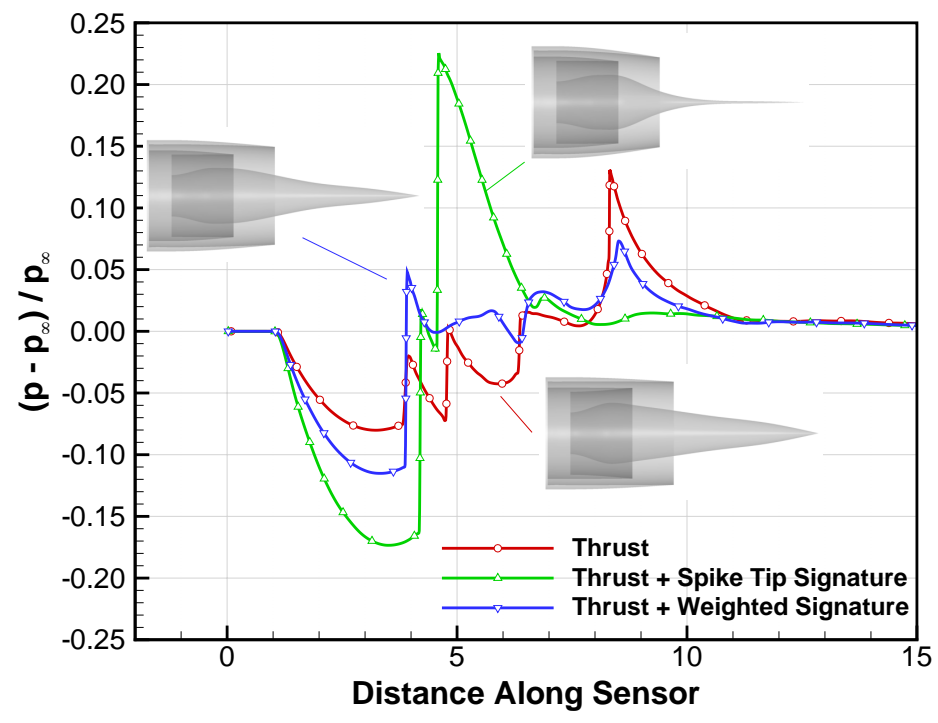

Figure 23: Nearfield pressure signatures generated by the three optimized nozzles.

In the final design optimization, we seek a balance between thrust maximization and the attenuation of both the nozzle lip shock and the conical shock by extending the sensor upstream and adjusting the weights in the objective function. Figure 22 shows the optimized shape and the corresponding flow solution. Comparing Figs. 22 and 21 reveals a significantly weaker nozzle lip shock and also a relatively weak conical shock at the spike tip. Thrust recovers to within $1.7 \%$ of the benchmark case. Figure 23 summarizes the pressure signatures obtained in each design optimization along with the optimized shapes. The results from the third optimization strike a balance between the first and second optimization problems.

\section{Conclusions}

We presented an adjoint consistent implementation of permeable boundary conditions that are well-suited for modeling propulsion-system effects in external-flow simulations. Numerical studies with mass-flow-rate outputs show that the adjoint solution near the permeable boundary is smooth, in particular for the $p_{\text {set }}$ subsonic outflow and $\left[T_{\mathrm{t}, \text { set }}, p_{\mathrm{t}, \mathrm{set}}\right]$ subsonic inflow boundary conditions. The two-shock wedge verification example demonstrates that mass-flow-rate outputs converge at $\mathcal{O}(h)$. The discretization error estimate for these outputs converges at the same rate, and the adaptation error indicator, which is a higher-order term, converges at a slightly higher rate. These observations match the expected results due to the non-smooth flowfield. The output convergence rate is primarily limited by the presence of shocks.

The results demonstrate the effectiveness of using mass-flow-rate outputs in analysis and design of lowboom, powered configurations. The low-boom aircraft example clearly shows that better results are obtained when mass-flow-rate outputs are included in the adaptive mesh refinement procedure - the mesh-convergence of this output is significantly accelerated without compromising the accuracy of the nearfield pressure signature. The first optimization example demonstrates the use of $p_{\text {set }}$ as a design variable to match a specified mass flow rate. We show simultaneous mass-flow-rate control for the main engine inlet and the upstream secondary flow paths. The final shape optimization problem demonstrates mass-flow-rate constraints together with a trade-off between the nearfield pressure-signature objective and the performance (thrust) objective. This example highlights new possibilities for shape optimization problems that are enabled with mass-flow-rate functionals and the permeable boundary conditions. In future work we plan to investigate adjoint consistency of more general outputs, for example flow distortion and total pressure recovery, in order to consider general propulsion-airframe integration problems. 


\section{Acknowledgements}

The authors gratefully acknowledge support from NASA's ARMD Commercial Supersonic Technology and LBFD/X-59 Projects. David Rodriguez was supported under NASA Ames Research Center contract NNA16BD60C. Computing resources were provided by the NASA High-End Computing Program. The authors thank Jeffrey Housman and Gaetan Kenway for reviewing the manuscript.

\section{References}

${ }^{1}$ Conners, T. R., Henne, P. A., and Howe, D. C., "A Method for Reducing Sonic Boom Strength by Tailoring the Shape of the Propulsive Streamtube," AIAA Paper 2013-2260, San Jose, CA, July 2013, 49th AIAA/ASME/SAE/ASEE Joint Propulsion Conference.

${ }^{2}$ Wintzer, M., Castner, R. S., and Geiselhart, K. A., "Airframe-Nozzle-Plume Interactions in the Context of Low Sonic Boom Design," AIAA Paper 2015-1045, Jan. 2015, 53rd AIAA Aerospace Sciences Meeting.

${ }^{3}$ Castner, R., Zaman, K., Fagan, A., and Heath, C., "Wedge Shock and Nozzle Exhaust Plume Interaction in a Supersonic Jet Flow," J. of Aircraft, Vol. 54, No. 1, 2017, pp. 125-134. doi:10.2514/1.C033623.

${ }^{4}$ Smith, S. C., Nemec, M., and Kris, S. E., "Integrated Nacelle-Wing Shape Optimization for an Ultra-High Bypass Fanjet Installation on a Single-Aisle Transport Configuration," AIAA Paper 2013-0543, Grapevine, TX, Jan. 2013, 51st AIAA Aerospace Sciences Meeting.

${ }^{5}$ Merle, A., Stück, A., and Rempke, A., "An Adjoint-based Aerodynamic Shape Optimization Strategy for Trimmed Aircraft with Active Engines," AIAA Paper 2017-3754, Denver, CO, June 2017, 35th AIAA Applied Aerodynamics Conference.

${ }^{6}$ Chiew, J. J. and Aftosmis, M. J., "Efficient Simulation of Multi-rotor Vehicles with Low Reynolds Number Propellers," AIAA Paper 2018-4119, Atlanta, GA, June 2018, AIAA Applied Aerodynamics Conference.

${ }^{7}$ Carlson, J.-R., "Inflow/Outflow Boundary Conditions with Application to FUN3D," NASA/TM-2011-217181, 2011, http://hdl.handle.net/2060/20110022658, visited Nov 2018.

${ }^{8}$ Kenway, G. K. and Kiris, C., "Aerodynamic shape optimization of the STARC-ABL concept for minimal inlet distortion," AIAA Paper 2018-1912, San Jose, CA, Jan. 2018, AIAA/ASCE/AHS/ASC Structures, Structural Dynamics, and Materials Conference.

${ }^{9}$ Rodriguez, D. L., "Multidisciplinary Optimization Method for Designing Boundary-Layer-Ingesting Inlets," J. of Aircraft, Vol. 46, No. 3, 2009, pp. 883-894. doi:10.2514/1.38755.

${ }^{10}$ Ordaz, I., Rallabhandi, S. K., Nielsen, E. J., and Diskin, B., "Mitigation of Engine Inlet Distortion through Adjoint-Based Design," AIAA Paper 2017-3410, Denver, CO, June 2017, 35th AIAA Applied Aerodynamics Conference.

${ }^{11}$ Gray, J. S. and Martins, J. R. R. A., "Coupled Aeropropulsive Design Optimization of a Boundary Layer Ingestion Propulsor," The Aeronautical Journal, Oct. 2018. doi:10.1017/aer.2018.120.

${ }^{12}$ Fidkowski, K. J. and Darmofal, D. L., "Review of Output-Based Error Estimation and Mesh Adaptation in Computational Fluid Dynamics," AIAA Journal, Vol. 49, No. 4, April 2011, pp. 673-694. doi:10.2514/1.J050073.

${ }^{13}$ Lee, B. J., Liou, M.-S., and Kim, C., "Optimizing a Boundary-Layer-Ingestion Offset Inlet by Discrete Adjoint Approach," AIAA Journal, Vol. 48, No. 9, 2010, pp. 2008-2016. doi:10.2514/1.J050222.

${ }^{14}$ Rodriguez, D. L., Aftosmis, M. J., and Nemec, M., "Formulation and Implementation of Inflow/Outflow Boundary Conditions to Simulate Propulsive Effects," AIAA Paper 2018-0334, Kissimmee, FL, Jan. 2018, AIAA SciTech Forum.

${ }^{15}$ Nemec, M. and Aftosmis, M. J., "Toward Automatic Verification of Goal-Oriented Flow Simulations," NASA/TM 2014218386, National Aeronautics and Space Administration, Center for AeroSpace Information, 7115 Standard Drive, Hanover, MD 21076, 2014, http://hdl.handle.net/2060/20150000864.

${ }^{16}$ Nemec, M. and Aftosmis, M. J., "Output Error Estimates and Mesh Refinement in Aerodynamic Shape Optimization," AIAA Paper 2013-0865, Grapevine, TX, Jan. 2013, 51st AIAA Aerospace Sciences Meeting.

${ }^{17}$ Park, M. A., Aftosmis, M. J., Campbell, R. L., Carter, M. B., Cliff, S. E., and Bangert, L. S., "Summary of the 2008 NASA Fundamental Aeronautics Program Sonic Boom Prediction Workshop," Journal of Aircraft, Vol. 51, No. 3, 2014 , pp. $987-1001$. doi:10.2514/1.C032589.

${ }^{18}$ Anderson, G., Aftosmis, M. J., and Nemec, M., "Cart3D Simulations for the Second AIAA Sonic Boom Prediction Workshop," AIAA Paper 2017-3255, June 2017, 35th AIAA Applied Aerodynamics Conference.

${ }^{19}$ Park, M. A. and Nemec, M., "Nearfield Summary and Statistical Analysis of the Second AIAA Sonic Boom Prediction Workshop," Journal of Aircraft, Vol. 0, No. 0, 2018, pp. 1-24. doi:10.2514/1.C034866, Article in Advance.

${ }^{20}$ van Leer, B., "Flux-Vector Splitting for the Euler Equations," ICASE Report 82-30, Sept. 1982.

${ }^{21}$ Aftosmis, M. J., Berger, M. J., and Adomavicius, G., "A Parallel Multilevel Method for Adaptively Refined Cartesian Grids with Embedded Boundaries," AIAA Paper 2000-0808, Reno, NV, Jan. 2000.

${ }^{22}$ Aftosmis, M. J., Berger, M. J., and Murman, S. M., "Applications of Space-Filling-Curves to Cartesian Methods for CFD," AIAA Paper 2004-1232, Reno, NV, Jan. 2004.

${ }^{23}$ Berger, M. J., Aftosmis, M. J., and Murman, S. M., "Analysis of Slope Limiters on Irregular Grids," AIAA Paper 2005-0490, Reno, NV, Jan. 2005.

${ }^{24}$ Pandya, S. A., Murman, S. M., and Aftosmis, M. J., "Validation and Inlet and Exhaust Boundary Conditions for a Cartesian Method," AIAA Paper 2004-4837, Providence, RI, Aug. 2004, 22nd AIAA Applied Aerodynamics Conference and Exhibit.

${ }^{25}$ Roache, P. J., "Verification of Codes and Calculations," AIAA Journal, Vol. 36, No. 5, May 1998, pp. 696-702.

${ }^{26}$ Venditti, D. A. and Darmofal, D. L., "Grid Adaptation for Functional Outputs: Application to Two-Dimensional Inviscid Flow," Journal of Computational Physics, Vol. 176, 2002, pp. 40-69. doi:10.1006/jcph.2001.6967. 
${ }^{27}$ Huang, A. C., Allmaras, S. R., Galbraith, M. C., and Darmofal, D. L., "Well-Posed Subsonic Inflow-Outflow Boundary Conditions for the Navier-Stokes Equations," AIAA Paper 2018-0361, Kissimmee, FL, Jan. 2018, 2018 AIAA Aerospace Sciences Meeting.

${ }^{28}$ Hicken, J. and Zingg, D., "Dual consistency and functional accuracy: a finite-difference perspective," Journal of Computational Physics, Vol. 256, 2014, pp. 161 - 182. doi:https://doi.org/10.1016/j.jcp.2013.08.014.

${ }^{29} \mathrm{Lu}$, J., An a posteriori Error Control Framework for Adaptive Precision Optimization using Discontinuous Galerkin Finite Element Method, Ph.D. thesis, Massachusetts Institute of Technology, 2005.

${ }^{30}$ Giles, M. B. and Pierce, N. A., "Adjoint equations in CFD - Duality, boundary conditions and solution behaviour," AIAA Paper 1997-1850, June 1997, 13th Computational Fluid Dynamics Conference.

${ }^{31}$ Jameson, A., "Aerodynamic design via control theory," NASA/CR 181749, National Aeronautics and Space Administration, Center for AeroSpace Information, 7115 Standard Drive, Hanover, MD 21076, 1988, ICASE-88-64.

${ }^{32} \mathrm{Berg}$, J. and Nordström, J., "Duality based boundary conditions and dual consistent finite difference discretizations of the Navier-Stokes and Euler equations," Journal of Computational Physics, Vol. 259, 2014, pp. 135 - 153. doi:https://doi.org/10.1016/j.jcp.2013.11.031.

${ }^{33}$ Gill, P. E., Murray, W., Michael, and Saunders, A., "SNOPT: An SQP algorithm for large-scale constrained optimization," SIAM Journal on Optimization, Vol. 12, 1997, pp. 979-1006.

${ }^{34}$ Nemec, M. and Aftosmis, M. J., "Adjoint Sensitivity Computations for an Embedded-Boundary Cartesian Mesh Method," Journal of Computational Physics, Vol. 227, 2008, pp. 2724-2742.

${ }^{35}$ Nemec, M. and Aftosmis, M. J., "Parallel Adjoint Framework for Aerodynamic Shape Optimization of Component-Based Geometry," AIAA Paper 2011-1249, Orlando, FL, Jan 2011, 49th AIAA Aerospace Sciences Meeting.

${ }^{36}$ Nemec, M., Aftosmis, M. J., and Wintzer, M., "Adjoint-Based Adaptive Mesh Refinement for Complex Geometries," AIAA Paper 2008-0725, Reno, NV, Jan. 2008.

${ }^{37}$ Wintzer, M., Nemec, M., and Aftosmis, M. J., "Adjoint-Based Adaptive Mesh Refinement for Sonic Boom Prediction," 26th AIAA Applied Aerodynamics Conference, No. 2008-6593, AIAA, Honolulu, HI, August 2008.

${ }^{38}$ Heath, C., Gray, J. S., Park, M. A., Nielsen, E. J., and Carlson, J.-R., "Aerodynamic Shape Optimization of a Dual-Stream Supersonic Plug Nozzle," AIAA Paper 2015-1047, Jan. 2015, 53rd AIAA Aerospace Sciences Meeting. 